\title{
Hydrogen-Bonded Complexes of Neutral Nitroxide Radicals with 2-Propanol Studied by Multifrequency EPR/ENDOR
}

\author{
Anton Savitsky ${ }^{1}$ (D) Anna Nalepa ${ }^{2} \cdot$ Taras Petrenko $^{3} \cdot$ Martin Plato $^{4}$. \\ Klaus Möbius ${ }^{4} \cdot$ Wolfgang Lubitz ${ }^{2}$ (i)
}

Received: 20 July 2021 / Revised: 7 October 2021 / Accepted: 9 October 2021 /

Published online: 10 December 2021

(c) The Author(s) 2021

\begin{abstract}
The hydrogen bond plays a key role in weak directional intermolecular interactions. It is operative in determining molecular conformation and aggregation, and controls the function of many chemical systems, ranging from inorganic, organic to biological molecules. Although an enormous amount of spectroscopic information has been collected about hydrogen-bond formation between molecules with closed-shell electronic configuration, the details of such interactions between open-shell radicals and closed-shell molecules are still rare. Here we report on an investigation of hydrogenbonded complexes between pyrroline-type as well as piperidine-type neutral nitroxide radicals and an alcohol, i.e., 2-propanol. These nitroxide radicals are commonly used as EPR spin labels and probes. To obtain information on the geometry of the complexes and their electronic structure, multi-resonance EPR techniques at various microwave frequencies (X-, Q-, W-band, $244 \mathrm{GHz}$ ) have been employed in conjunction with DFT calculations. The planar five-membered ring system of the pyrrolinetype nitroxide radical was found to form exclusively well-defined in-plane $\sigma$-type hydrogen-bonded complexes with one 2-propanol molecule in the first solvation shell in frozen solution. The measured hyperfine parameters of the hydrogen-bridge proton and the internal magnetic parameters describing the electron Zeeman and the electron-nuclear hyperfine and nuclear quadrupole interactions are in good agreement with values predicted by state-of-the-art DFT calculations. In contrast, multiresonance EPR on the non-planar six-membered ring system of the piperidine-type nitroxide radical (TEMPOL) reveals a more complex situation, i.e., a mixture of a $\sigma$-type with, presumably, an out-of-plane $\pi$-type complex, both present in comparable fraction in frozen solution. For TEMPOL, the DFT calculations failed to predict magnetic interaction parameters that are in good agreement with experiment, apparently due to the considerable flexibility of the nitroxide and hydrogen-bonded complex. The detailed information about nitroxide/solvent complexes is of particular
\end{abstract}

Dedicated to Prof. Kev Salikhov on the occasion of his 85th birthday.

Extended author information available on the last page of the article 
importance for Dynamic Nuclear Polarization (DNP) and site-directed spin-labeling EPR studies that employ nitroxides as polarizing agents or spin labels, respectively.

\section{Introduction}

Weak hydrogen bonding is the most important directional intermolecular interaction in chemistry [1]. It is operative for determining molecular conformation and aggregation, as well as for the functionality of many chemical systems, ranging from inorganic, organic to biological molecules [2-7]. Since its discovery more than 100 years ago, the hydrogen bond is still a topic of vital research in diverse scientific branches, including mineralogy, material science, inorganic and organic chemistry, supramolecular chemistry, biochemistry, molecular biology and medicine, as well as molecular-design based pharmacology. In his seminal book, "The Nature of the Chemical Bond", Linus Pauling gives credit to T. S. Moore and T. F. Winmill, who first mentioned the hydrogen bond in 1912 [8, 9].

Although an enormous amount of spectroscopic information has been collected about hydrogen-bonded complexes between closed-shell molecules [10], details (in particular geometry) of the hydrogen-bonded complexes between closed-shell and open-shell molecules, such as reactive short-lived radicals, are still rare. Well-established knowledge is, however, that weak hydrogen-bonding effects are important in significantly modulating free radical properties. As an example, quinone radicals are known to markedly change their photochemical, kinetic, and spectroscopic properties upon formation of a hydrogen-bonded complex that involves the oxygen atom and retains an unpaired electron. Both neutral quinone radicals and radical ions are frequent intermediates in quinone-mediated redox processes. The hydrogen-bond situation of the quinone cofactors in the bacterial photosynthetic reaction center [11-17], in plant Photosystem I [18-21] and Photosystem II [22] was clarified in recent years. Moreover, photosynthetic and related model systems were investigated in great detail both experimentally and theoretically [23-26] making the hydrogenbonded complexes of quinone radicals with organic ligands the best understood $\mathrm{H}$-bond system in molecular biology of today.

In contrast to the quinone radicals, hydrogen-bond formation of other important radicals, such as nitroxide radicals, is still under controversial debate. Numerous experimental EPR [27, 28], NMR [29-40], IR [41, 42], X-ray [43-45], calorimetry $[42,46]$ investigations on this issue have been published in the past. Detailed information on hydrogen bonds of nitroxide radicals was gained from liquid-solution NMR studies [29-40], for instance for hydrogen bonds of nitroxide radicals with alcohols, phenols, amines, acids, hydroperoxides and water. From the analysis of the paramagnetic contact shifts of donor molecules and their relaxation properties and the electron spin densities of hydrogen-bonded complexes were obtained.

As to EPR, the magnetic interaction parameters of nitroxide radicals - the electron Zeeman, the electron-nuclear hyperfine and quadrupole interactions-depend markedly on the radical microenvironment, i.e., on the nature of the matrix in which the radicals are dissolved. Already early EPR studies of stable nitroxide radicals in the liquid phase have recognized the isotropic hyperfine couplings and $g$ values to 
Scheme 1 Molecular structures of the pyrroline-type (R1) and piperidine-type (R2) nitroxide radicals used in this work
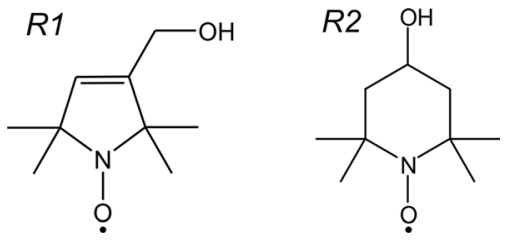

be sensitive probes of the polarity and proticity of the medium in which the radicals are embedded [47-49]. Later studies, including high-field EPR, have demonstrated the extraordinary sensitivity of the principal ${ }^{14} \mathrm{~N} A_{z z}$ hyperfine and $g_{x x}$ Zeeman tensor components on the local microenvironment of the nitroxides both in homogeneous frozen solutions and nitroxide spin-labeled proteins [50-57]. Several theoretical attempts were made to understand the correlations of such interaction parameters with polarity and proticity characteristics of the microenvironment [57-62].

Recently, we have demonstrated that for nitroxide radicals in frozen protic solvents and spin-labeled proteins, a pronounced heterogeneity of $g_{x x}, A_{z z}$ and also $P_{z z}$ $\left({ }^{14} \mathrm{~N}\right.$ quadrupole tensor component) is present that can be resolved when using highfield cw EPR and ELDOR-detected NMR techniques [53, 54, 56, 63]. The distinct correlation of $g_{x x}, A_{z z}$ and $P_{z z}$ values senses different nitroxide populations that are characterized by their different local environments. These differences in environments were tentatively ascribed to a different hydrogen-bond situation of the nitroxides, i.e., whether they develop one or two H-bonds to the solvent molecules - or no $\mathrm{H}$-bonds at all. An exact assignment of the various nitroxide $\mathrm{H}$-bond situations requires, however, an experimental and theoretical characterization of the hydrogenbonded complexes. In particular, the hyperfine coupling parameters of the hydrogen in the hydrogen bond are required, from which the geometrical structure of the complexes can be derived.

It this work, we report on multi-frequency, multi-resonance EPR investigations of two types of hydrogen-bonded complexes with 2-propanol. They consist of two inherently different types of nitroxide radicals: $(i)$ the five-membered ring system of a pyrroline-type nitroxide radical, and (ii) the six-membered ring system of a piperidine-type nitroxide, see Scheme 1. These nitroxides often serve as models for the MTS spin label and the non-natural amino-acid TOAC spin label, respectively, both are widely used in side-directed spin labeling (SDSL) studies (see, for instance, overview articles $[64,65])$. The aim is to elucidate the structure of hydrogen-bonded complexes. Several structural models of hydrogen-bonded nitroxide-alcohol complexes have been proposed previously, see for instance [66, 67]. They include two types of the models: (i) an in-plane $\sigma$-type model in which the $\mathrm{H}$-bond is placed in the $\sigma$-orbital plane of the oxygen and directed toward an oxygen lone-pair orbital (with an $\mathrm{N}-\mathrm{O}-\mathrm{H}$ bond angle of $\sim 120^{\circ}$ ), and (ii) an out-of-plane $\pi$-type model in which the orbital of the hydrogen-bonded proton contacts the $p_{z}$ orbital of either oxygen, $\pi(\mathrm{O})$, or nitrogen, $\pi(\mathrm{N})$, of the nitroxide atoms.

At first, we specify an experimental methodology that is able to provide highly detailed information about the hydrogen-bonded complex. We have applied various EPR techniques on the nitroxide R1, employing continuous wave (cw) EPR, 
hyperfine sublevel correlation (HYSCORE) and electron nuclear double resonance (ENDOR) at different microwave frequencies. After having found the optimal EPR technique for this purpose, the second nitroxide radical, R2, was investigated to understand the differences in the hydrogen bonding situations of these spin probes and to explain existing discrepancies of the NMR and EPR results reported earlier. The obtained spectroscopic information is compared with predictions calculated by density functional theory (DFT).

\section{Theory}

\subsection{Spin Hamiltonian}

For a nitroxide radical $(\mathrm{S}=1 / 2)$, the static spin Hamiltonian, $\hat{H}$, that describes the time-independent spin interactions, consists of the terms

$$
\frac{\hat{H}}{h}=\frac{\mu_{B}}{h} \cdot \boldsymbol{B}_{0} \cdot \tilde{g} \cdot \hat{S}-\sum_{i} \frac{g_{n i} \cdot \mu_{N}}{h} \cdot \boldsymbol{B}_{0} \cdot \hat{I}_{i}+\sum_{i} \hat{S} \cdot \tilde{A}_{i} \cdot \hat{I}_{i}+\sum_{i} \hat{I}_{i} \cdot \tilde{P}_{i} \cdot \hat{I}_{i}
$$

where $\widehat{H}$ contains the field-dependent electron and nuclear Zeeman interactions as well as the field-independent electron-nuclear hyperfine and nuclear quadrupole interactions with magnetic nuclei. For the quadrupole interaction to exist, nuclei with $I>1 / 2$ in an asymmetric electronic environment are required. $\boldsymbol{B}_{0}$ is the external magnetic field (a vector), $\tilde{g}, \tilde{A}$ and $\tilde{P}$ are the corresponding interaction tensors (here represented by $3 \times 3$ matrices) of the electron Zeeman, hyperfine and quadrupole interactions $\left(h\right.$ : Planck constant; $\mu_{B}, \mu_{N}$ : Bohr and nuclear magnetons; $g_{n}$ : nuclear g-factors; $\widehat{S}, \widehat{I}$ : electron and nuclear spin vector operators; the summation is over all nuclei of the system).

In disordered solids (frozen solutions), in which the motion of the nitroxide molecules is substantially restricted, the line shape of the EPR spectrum at low external magnetic fields is governed by the field-independent nitrogen electron-nuclear hyperfine interaction (characterized by the A-tensor components, $I\left({ }^{14} \mathrm{~N}\right)=1$ ). At high external magnetic fields, the EPR spectrum is governed by the combined effect of the electron spin and orbital Zeeman interactions as well as the spin-orbit interaction (determining the magnitude of the g-tensor components). Both hyperfine and g-tensors are sensitive to the molecular structure and molecular axes system of the nitroxide, see Fig. 1a. The spin interaction terms of the unpaired electron with other magnetic nuclei in the system are small and typically not resolved in the EPR spectrum but are observed in the ENDOR spectra.

For a proton in a typical hydrogen bond to the nitroxide, the hyperfine tensor $\tilde{A}$ is composed of isotropic (scalar) and anisotropic (dipolar) contributions: $\tilde{A}=a_{i s o} \cdot \tilde{1}+\tilde{A}_{d i p}$, where $\tilde{1}$ is the unity tensor (equal to the unity matrix).

The isotropic part, $a_{i s o}$, is expected to be small compared to the anisotropic term, $\tilde{A}_{d i p}$, which arises from the dipolar interaction between the unpaired electron and the proton. In the simple point-dipole approximation (only the unpaired electron 

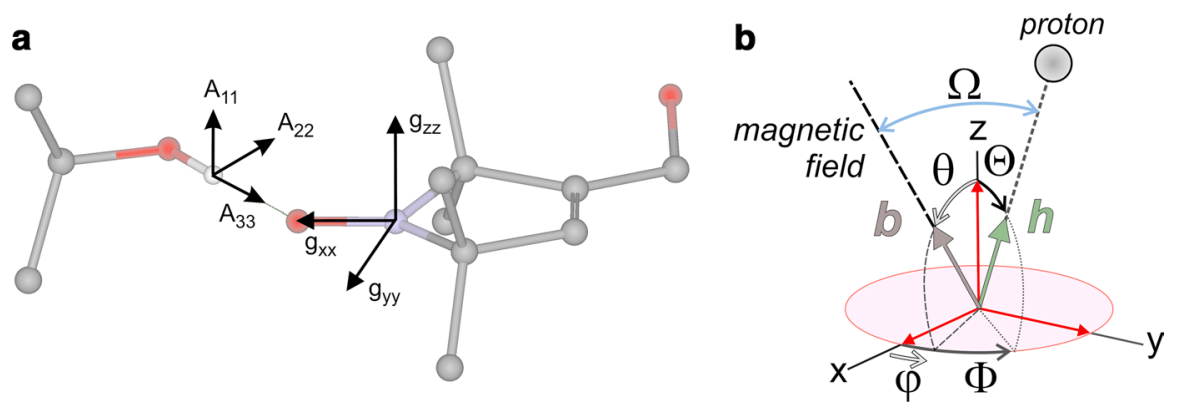

Fig. 1 a Orientation of the nitroxide g-tensor and the bridging hydrogen hyperfine tensor in the molecular frame of the $\sigma$-type R1/2-propanol complex. The irrelevant protons are omitted for clarity. b Definition of the hydrogen-bonded complex geometry in the g-tensor frame of the nitroxide radical R1 (with the oxygen atom as origin of the frame). The unity vectors $\boldsymbol{b}$ and $\boldsymbol{h}$ show the directions of the external magnetic field $B_{0}$ and the dipolar $\mathrm{O} \cdots \mathrm{H}$ hydrogen-bond axis whose positioning in the nitroxide g-tensor frame is determined by polar angles $(\theta, \varphi)$ and $(\Theta, \Phi)$, respectively. The angle between $\boldsymbol{b}$ and $\boldsymbol{h}$ vectors is given by $\Omega$

spin density on the nitroxide oxygen is considered), the observed hyperfine splitting by the anisotropic interaction (in frequency units) is given by

$$
A_{\text {dip }}=\frac{\mu_{0}}{4 \pi h} \cdot \frac{g_{e} \cdot g_{n}(H) \cdot \mu_{B} \cdot \mu_{N}}{r^{3}} \cdot \rho_{O} \cdot\left(3 \cos ^{2} \Omega-1\right)=T \cdot\left(3 \cos ^{2} \Omega-1\right)
$$

where $\rho_{\mathrm{O}}$ is the unpaired electron spin density at the oxygen, $\Omega$ is the angle between the applied magnetic field and the direction of the hydrogen bond, Fig. 1b, and $r$ is the H-bond length. For the proton in the hydrogen bond, the principal dipolar interaction value is given by $T=79.2 \cdot \rho_{O} \cdot\left(r / r_{0}\right)^{3} \mathrm{MHz}$ when choosing $r_{0}=0.1 \mathrm{~nm}$. For deuterons, the numerical pre-factor 79.2 for protons is replaced by 12.16 according to the ratio of the nuclear $g$ values. The dipolar ${ }^{1} \mathrm{H}$ hyperfine tensor $\left[A_{11}, A_{22}, A_{33}\right]$ resulting from this point dipolar approximation is axially symmetric, with the principal components $A_{33}=A_{\|}=+2 T$ for $\Omega=0$ (direction along the hydrogen bond) and $A_{11}=A_{22}=-T$ for $\Omega=90^{\circ}$. For the nitroxide, non-axial symmetry $\left(A_{11} \neq A_{22}\right)$ of the dipolar ${ }^{1} \mathrm{H}$-hyperfine tensor is expected due to contributions of dipolar hyperfine interaction with substantial unpaired electron spin density on the nitroxide nitrogen atom, see below.

For hydrogen-bonded deuterium, the nuclear quadrupole interaction between the nuclear electric quadrupole moment, $Q$, and an electric field gradient at the deuterium nucleus is described by the quadrupole tensor, $\tilde{P}$. In its principal axes system, the quadrupole tensor is traceless. Its largest principal value is $P_{33}=e^{2} \cdot q \cdot Q / 2 h$, where $e q$ is the electric field gradient of the electron plus the nuclear charge distribution along this direction, and $\eta=\left(P_{11}-P_{22}\right) / P_{33}$ is the asymmetry parameter of the charge distribution, with the convention $\left|P_{33}\right|>\left|P_{22}\right|>\left|P_{11}\right|$ and $0 \leq \eta \leq 1[68$, 69]. The largest principal value of the quadrupole tensor is approximately along the direction of the H-bond. The field gradient is related to the distribution of electrical charges around the nucleus and thus related to the specific bonding geometry. Therefore, its value can be used to infer information on the hydrogen bond. 


\subsection{Molecular Structure and Axes System of Nitroxide Radical}

The magnetic interactions of the nitroxide radical are related to its molecular structure. It is common practice to determine the nitroxide g-tensor directions $\boldsymbol{x}, \boldsymbol{y}, \boldsymbol{z}$ (and the principal values $\left.\left[g_{x x}, g_{y y}, g_{z z}\right]\right)$ within the nitroxide molecular structure as X-axis directed along the NO bond, z-axis along the nitrogen $2 \mathrm{p}_{\mathrm{z}}$ orbital, and y-axis perpendicular to the $\mathrm{x}-\mathrm{z}$ plane. The principal directions of the ${ }^{14} \mathrm{~N}$ hyperfine and quadrupole tensors, $\left[A_{x x}, A_{y y}, A_{z z}\right]$ and $\left[P_{x x}, P_{y y}, P_{z z}\right]$, are supposed to be collinear with the corresponding g-tensor axes. The main component of the hyperfine tensor of a bridging hydrogen/deuteron, $A_{33}$, is to good approximation directed along the H(D)bond direction. The $A_{11}$, is perpendicular to the $\mathrm{COH}(\mathrm{D})$ plane of the 2-propanol. The eigenvector of the largest $\mathrm{D}$ nuclear quadrupole tensor component $P_{33}$ is also expected to be oriented along the D-bond direction to first approximation, while the $P_{11}$ and $P_{22}$ directions are approximately collinear with the corresponding hyperfine tensor directions.

\section{Materials and Methods}

\subsection{Materials and Sample Preparation}

The nitroxide radicals 3-hydroxymethyl-2,2,5,5-tetramethylpyrrolin-1-oxyl in their protonated (abbreviated as R1 throughout the text) and perdeuterated $\left(\mathrm{R} 1-\mathrm{D}_{16}\right)$ form were synthesized as described previously [70]. The nitroxide radical 4-hydroxy2,2,6,6-tetramethylpiperidine-1-oxyl (TEMPOL, abbreviated as R2 throughout the text) in its perdeuterated form $\left(\mathrm{R} 2-\mathrm{D}_{17}(\mathrm{OH})\right.$ ) was used as purchased (Aldrich, 97\% $\mathrm{D}, 95 \%)$. The solvents 2-propanol- $\mathrm{H}_{8}, 2$-propanol- $\mathrm{D}_{1}(\mathrm{OD})$ and 2-propanol- $\mathrm{D}_{7}(\mathrm{OH})$ were obtained from Aldrich in their purest commercially available form and used without further purification.

The nitroxide radicals were directly dissolved in 2-propanol to obtain the concentration of $1 \mathrm{mM}$. The sample solutions were filled into quartz capillaries (I.D. $=3.8 \mathrm{~mm}$ for X-band EPR; I.D. $1.8 \mathrm{~mm}$ for Q-band and $244 \mathrm{GHz} \mathrm{EPR}$; I.D. $=0.6 \mathrm{~mm}$ for $\mathrm{W}$-band EPR and I.D. $=4 \mathrm{~mm}$ for NMR) and shock frozen in liquid nitrogen to form a glass prior to the transfer into the precooled EPR probehead.

\subsection{EPR and NMR Experiments}

X-Band (9.5 GHz, 0.34 T) cw (continuous wave) and pulsed EPR measurements were performed using a Bruker ELEXSYS 580 spectrometer equipped with an Oxford Instruments ESR 935 cryostat, ITC4 temperature controller and pulsed ENDOR resonator (Bruker, EN 4118X-MD4). For cw EPR experiments, a fieldmodulation frequency of $100 \mathrm{kHz}$ and modulation amplitude of $50 \mu \mathrm{T}$ were used. The incident microwave power was adjusted to avoid any saturation effects.

Q-band (34 GHz, 1.21 T) cw EPR measurements were carried out using a Bruker ER200E spectrometer equipped with a Bruker ER051QG microwave (mw) 
bridge and a home-built $\mathrm{TE}_{011}$ Q-band cavity [71]. A field-modulation frequency of $100 \mathrm{kHz}$ and modulation amplitude of $50 \mu \mathrm{T}$ were used. Pulsed Q-band EPR experiments were carried out on a Bruker ELEXSYS E580 spectrometer with a Super Q-FT mw bridge and Oxford-CF935 liquid helium cryostat, ITC-503 temperature controller and a home-built ENDOR cavity similar to that used for $\mathrm{cw}$ EPR measurements.

W-band (95 GHz, $3.4 \mathrm{~T}$ ) EPR and ESEEM experiments were performed with a laboratory-built W-band spectrometer operating both in $\mathrm{cW}$ and pulsed mode, as described previously $[72,73]$. The temperature of the sample was controlled by a temperature stabilized nitrogen gas-flow cryostat in the probe-head. All cw EPR experiments were performed using a gold plated bronze $\mathrm{TE}_{011}$ resonator. Low mw power $(<1 \mu \mathrm{W})$ incident on the critically coupled loaded cavity $\left(\omega_{1}<1.5 \cdot 10^{5} \mathrm{rad} \cdot \mathrm{s}^{-1}\right)$ was chosen to avoid saturation of the sample. The external field was modulated at $8 \mathrm{kHz}$ with an amplitude of $50 \mu \mathrm{T}$. For ${ }^{14} \mathrm{~N}$-ESEEM experiments, spectrometer settings were used identical to those described previously [70]. For W-band ENDOR experiments, we used a modified W-band EPR spectrometer (Bruker ELEXSYS E680) operating at about $94 \mathrm{GHz}$ [63] equipped with a helium flow cryostat. The ENDOR experiments were carried out using two home-built mw cavities which contained a radiofrequency (RF) solenoid of Teflon-coated silver wire integrated into a commercial W-band ENDOR probe-head (Bruker). The RF coil had either 7 turns or 21 turns for optimized performance at low RF frequencies (<100 MHz, optimum performance at $20 \mathrm{MHz}$, which corresponds to the deuterium Larmor frequency) or high RF frequencies ( $>100 \mathrm{MHz}$, optimum performance at $140 \mathrm{MHz}$, which corresponds to the proton Larmor frequency), respectively. The optimal RF $\pi$-pulse length of about $20 \mu$ s is obtained for both deuterons and protons using $100 \mathrm{~W}$ output power of the RF amplifier. To ensure broadband mw excitation and minimize signal distortions caused by stray fields from the high-power RF excitation, the loaded quality factor was lowered to 700 to obtain a microwave frequency bandwidth of $130 \mathrm{MHz}$.

$244 \mathrm{GHz}$ cw EPR experiments were performed using a home-built EPR spectrometer equipped with a non-resonant EPR probehead described previously [74]. A field-modulation frequency of $100 \mathrm{kHz}$ and modulation amplitude of $50 \mu \mathrm{T}$ and 0.2 mT were used.

All multi-frequency cw EPR and pulsed EPR experiments were performed at $90 \mathrm{~K}$ and $60 \mathrm{~K}$, respectively, well below the 2-propanol glass transition temperature of $T_{g}=121 \mathrm{~K}[75,76]$. The spectra analysis and simulation procedures were performed on the basis of the EasySpin toolbox for the Matlab program package [77].

NMR experiments were performed using a Bruker Ascend $400 \mathrm{MHz}$ NMR spectrometer.

\subsection{DFT Computational Methods}

Calculations of the local hyperfine and quadrupole tensors as well as of the global g-tensor of the hydrogen-bonded radicals were performed on the level of density functional theory (DFT) using the quantum-chemical program package ORCA [78]. 
The actual hyperfine-, quadrupole- and g-tensor calculations were preceded by an ORCA geometry optimization procedure to establish minimum energy molecular structures of the two radical species, $\mathrm{R}_{1}$ and $\mathrm{R}_{2}$. Normally, these calculations were performed on molecules in the gas-phase, but additionally, in selected cases, solvent effects were included (see below). No geometrical restraints were introduced. Starting geometries were obtained by a Molecular Modeling software on a semiempirical level.

ORCA input settings for the geometry optimizations and for the calculation of the g- and hyperfine/quadrupole tensors $\tilde{g}, \tilde{A}$ and $\tilde{P}$ were chosen as follows:

I. Geometry optimizations: Spin unrestricted SCF (Kohn-Sham theory), standard DFT functional B3LYP and basis set def2-TZVP (Triple zeta valence basis set with "new" polarization functions, developed by the Ahlrichs group [79]).

II. g-tensor: DFT settings same as in (i), but including the $\operatorname{SOMF}(1 \mathrm{X})$ treatment for the spin-orbit coupling and an energy window of -100 to +100 a.u. for the contribution of molecular excited states.

III. A- and P-tensors: Same as in (i) and (ii) with a very fine integration, grid "5" for high numerical accuracy close to the nuclear regions.

The ORCA output provides isotropic and anisotropic (traceless) contributions to all three tensors, $\tilde{g}, \tilde{A}, \tilde{P}$, in the geometry optimized systems of the nitroxide/2-propanol complexes. Whereas g appears as a single global quantity, $A$ and $P$ are derived as local quantities for all relevant nuclear positions.

Since all tensors are initially calculated in an arbitrarily chosen laboratory system, they finally have to be transformed to the principal axes system $T_{g, l a b}$ of the g-tensor. These axes define the selected magnetic field orientations in the EPR experiments. Such transformations are easily performed by unitary transformations, e.g., $\tilde{X}_{g}=T_{g, l a b} \cdot \tilde{X}_{l a b} \cdot T_{g, l a b}^{-1}$, where $\tilde{X}$ stands for any interaction tensor at any nuclear position.

\section{Results and Discussion}

\subsection{Nitroxide R1, the Five-Membered Ring System}

We start the results and discussion section with the five-membered ring system, the nitroxide radical $\mathrm{R} 1$.

\subsubsection{Multi-frequency cw EPR Experiments}

Figure 2 shows multi-frequency cw EPR spectra of the R1-D ${ }_{16}$ nitroxide at $90 \mathrm{~K}$ in frozen 2-propanol- $\mathrm{D}_{8}$ solution. The spectra exhibit the typical powder pattern line shapes for a dilute random distribution of nitroxides at different mw frequencies and corresponding Zeeman fields. At X-band, the spectrum is dominated by the ${ }^{14} \mathrm{~N}$ nitrogen hyperfine interaction. The Q-band spectrum represents 


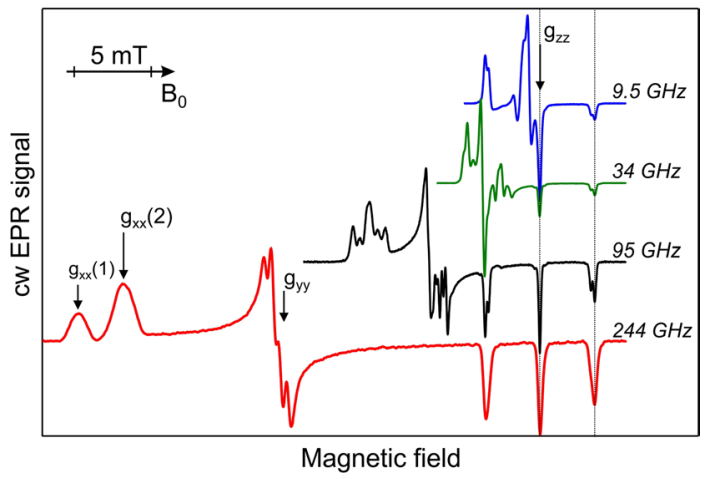

Fig. 2 Experimental multi-frequency cw EPR spectra of $1 \mathrm{mM} \mathrm{R1-D_{16 }}$ nitroxide in frozen 2-propanol- $\mathrm{D}_{8}$ solution at $90 \mathrm{~K}$. The spectra are aligned according to the magnetic field position corresponding to the principal $g_{z z}$ value of the g-tensor. In the $244 \mathrm{GHz}$ EPR spectrum in the $g_{x x}$ region two fractions (subensembles with different microenvironment) of the nitroxide radicals are resolved which are characterized by $g_{x x}(1)$ and $g_{x x}(2)$

an intermediate regime with comparable Zeeman $(g)$ and nitrogen hyperfine $(A)$ interaction contributions. The W-band EPR spectrum is dominated by the electron Zeeman interaction. It appears to be almost fully resolved exhibiting nitrogen hyperfine splittings in all spectral regions that are determined by canonical principal g-tensor values. The hyperfine resolution is completely ( $g_{x x}$ region) and partially ( $g_{y y}$ region) lost in the spectrum recorded at $244 \mathrm{GHz}$ due to a significant distribution of the $g_{x x(y y)}$ values typical for the so-called "g-strain" effect observed at higher Zeeman fields. In all frequency bands, the EPR spectra consist of two spectral contributions from sub-ensembles (fractions (1) and (2)) stemming from nitroxide fractions that are exposed to different microenvironments. These spectral contributions are characterized by two distinct sets of magnetic parameters, i.e., g- and A-tensor values.

The difference in $A_{z z}$ values manifests itself in an additional splitting of the $g_{z z}, M_{I}=-1$ hyperfine line which is observed in all microwave bands. This splitting is not clearly visible in the $244 \mathrm{GHz}$ spectrum depicted in Fig. 2 due to the high-field-modulation amplitude used. It can, however, be resolved using appropriate experimental settings (see Fig. S1 in SI).

The difference in $g_{x x}$ values results in an additional splitting in the $g_{x x}$ spectral region which is most prominent at $244 \mathrm{GHz}$. The combined analysis of the multifrequency EPR spectra yields the complete sets of g- and A-tensor principal values; they agree with those obtained from the analysis of W-band cw EPR spectra we reported previously [63], see Table 1 . The spectral intensities of the two contributions yield the relative weights of the nitroxide subensembles (1) and (2) of $0.40 \pm 0.02$ and $0.60 \pm 0.02$, respectively. It is noted that neither sample preparation nor freezing conditions influence the fractional weights.

Additionally, the distribution of the $g_{x x}$-tensor values in both nitroxide fractions, (1) and (2), is deduced from analysis of the $244 \mathrm{GHz}$ spectrum: The Gaussian FWHM distribution widths of $\delta g_{x x}(1)=1.9 \cdot 10^{-4}$ and $\delta g_{x x}(2)=2.1 \cdot 10^{-4}$ are equal 
Table 1 Magnetic parameters of $\mathrm{R} 1-\mathrm{D}_{16}$ nitroxide radicals in frozen 2-propanol- $\mathrm{D}_{8}$ and ortho-terphenyl (OTP) solutions as obtained from the analysis of the multi-frequency cw EPR, W-band ESEEM and W-band EDNMR spectra

\begin{tabular}{lllll}
\hline & $\begin{array}{l}{ }^{14} \mathrm{~N}\left[A_{x x}, A_{y y}, A_{z z}\right] / \\
\mathrm{MHz}\end{array}$ & $\begin{array}{l}{\left[g_{x x}, g_{y y}, g_{z z}\right]} \\
-2 / 10^{-6}\end{array}$ & $\begin{array}{l}{ }^{14} \mathrm{~N}\left[P_{x x}, P_{y y}, P_{z z}\right] / \\
\mathrm{MHz}\end{array}$ & Fraction \\
\hline 2-propanol (1) & $12.07 ; 12.07 ; 93.16$ & $9109 ; 6196 ; 2185$ & $+1.26 ;+0.51 ;-1.77$ & 0.4 \\
2-propanol (2) & $13.24 ; 13.30 ; 99.68$ & $8432 ; 6062 ; 2185$ & $+1.23 ;+0.39 ;-1.62$ & 0.6 \\
ortho-terphenyl* & $12.10 ; 12.18 ; 94.67$ & $9050 ; 6150 ; 2198$ & $+1.26 ;+0.48 ;-1.74$ & 1 \\
\hline
\end{tabular}

*The magnetic parameters for OTP/benzene are adopted from our previous studies [63, 70, 80]
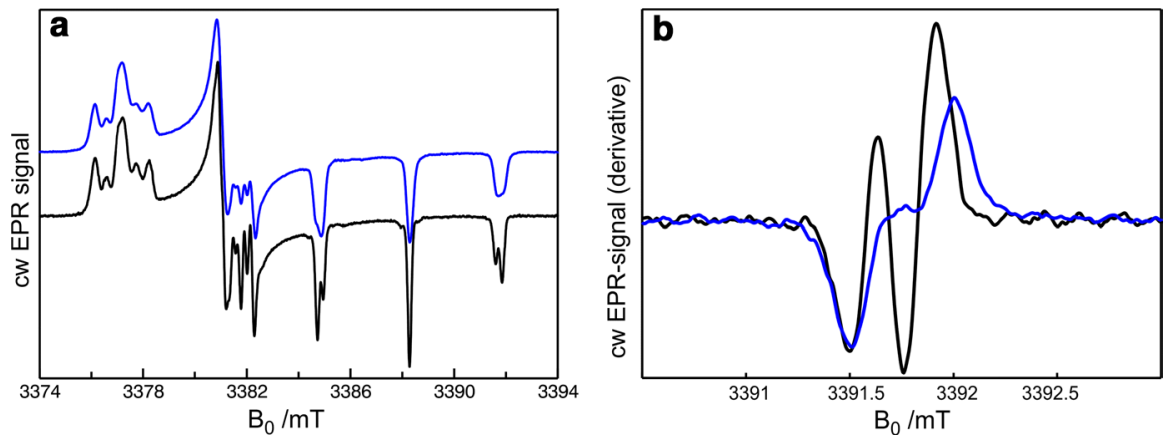

Fig. 3 a Experimental W-band cw EPR spectra of $1 \mathrm{mM}$ R1-D ${ }_{16}$ nitroxide in frozen 2-propanol- $\mathrm{D}_{8}$ (black trace) and 2-propanol- $\mathrm{D}_{7}(\mathrm{OH})$ (blue trace) solutions at $90 \mathrm{~K}$. b The first-derivative of $\mathrm{cw}$ EPR spectra in the $g_{z z}, M_{I}=-1$ spectral region (color figure online)

within experimental error of $\pm 1 \cdot 10^{-5}$ (see Fig. S2b in SI). Thus, the microenvironment of both nitroxide fractions appears to be equally well defined, which is in agreement with our previous study [63].

The cw EPR spectra shown in Fig. 2 do not allow to infer the nature of the two nitroxide subensembles in frozen 2-propanol, which differ only in the hydrogenbond situation of the nitroxide radical with respect to the solvent molecules. A firm assignment can only be made indirectly by comparing the magnetic parameters of both fractions with those obtained in a nonpolar, aprotic matrix such as orthoterphenyl (OTP)/benzene matrix [63], see Table 1. However, additional cw EPR experiments provide supporting insights: Fig. 3 compares the W-band cw EPR spectra of nitroxides recorded in 2-propanol- $\mathrm{D}_{8}$ and 2-propanol- $\mathrm{D}_{7}(\mathrm{OH})$, respectively. Changes are observed in the $g_{z z}$ spectral range. Hypothetically, they could be interpreted as an equilibrium shift between the nitroxide fractions toward one subensemble with smaller $A_{z z}$ value. This, however, would be in contradiction to the fact that the EPR spectra are in very good agreement in the $g_{x x}$ region. The comparison of the $g_{z z}, M_{I}=-1 \mathrm{cw}$ EPR line in derivative form, Fig. 3b, shows that only the line with higher $A_{z z}$ values is affected. The changes stem from an additional splitting caused by hyperfine interaction with the $\mathrm{OH}$ proton, which is close to the difference 

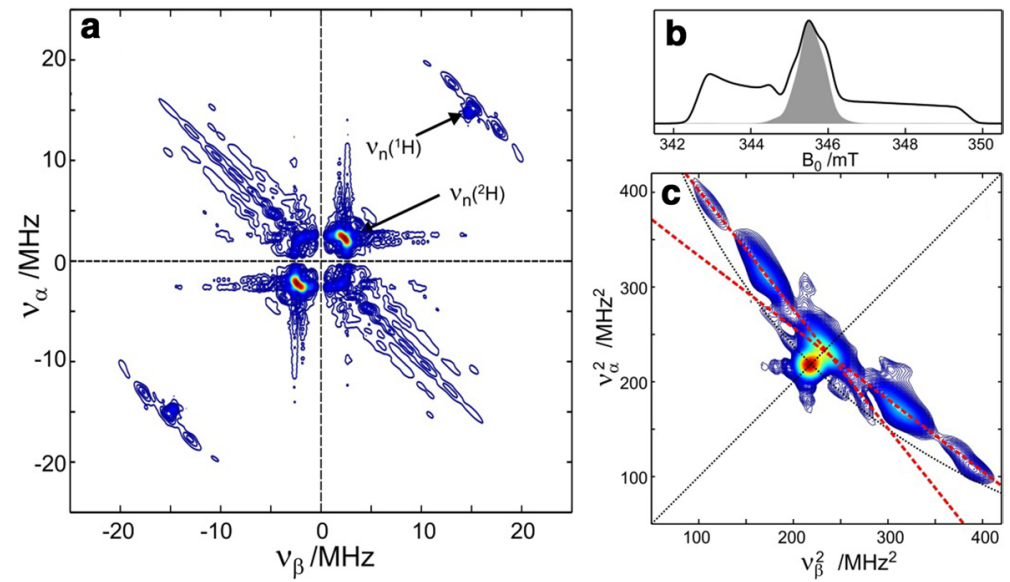

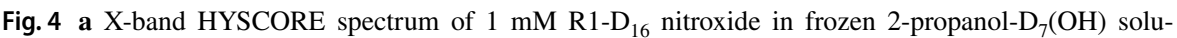
tion at $60 \mathrm{~K}$. b Field-swept echo-detected EPR spectrum with the magnetic field position and microwave excitation band width for HYSCORE recording indicated. $\mathbf{c}$ Extended view of the HYSCORE spectrum around the ${ }^{1} \mathrm{H}$ Larmor frequency in squared-frequency axes representation

between both $A_{z z}$ values. The analysis of Q- and W-band spectra $\left(g_{z z}, M_{I}=-1\right.$ line $)$ yields for this proton a hyperfine splitting of $4.5 \pm 0.3 \mathrm{MHz}$ (see Fig. S3 in SI). Thus, the nitroxide faction (2) must be assigned to nitroxides forming a hydrogen bond to the solvent.

At this point, cw EPR spectroscopy has exhausted its possibilities for structure determination because it is not capable to provide, to first order, exact ${ }^{14} \mathrm{~N}$ quadrupole values, $P_{i i}$, for both nitroxide subensembles, (1) and (2). Hence, additional W-band ESEEM experiments were performed to obtain $P_{x x}$ principal values (see Fig. S4 in SI). In combination with our recent results from ELDOR-detected NMR experiments in which exact $P_{z z}$ were measured [63], all quadrupole principal values for both fractions were obtained, see Table 1.

\subsubsection{X-band HYSCORE Experiments}

Figure 4a shows an X-band HYSCORE spectrum of R1-D 16 in 2-propanol- $\mathrm{D}_{7}(\mathrm{OH})$ recorded at the Zeeman field position indicated in Fig. $4 \mathrm{~b}$ at $60 \mathrm{~K}$. The spectrum is dominated by the deuterium spectral contributions centered around the D nuclear Larmor frequency of $2.2 \mathrm{MHz}$ in the $(-,-)$ and $(+,+)$ frequency quadrants. Additional signals appear around the ${ }^{1} \mathrm{H}$ nuclear Larmor frequency in the range between 10 and $20 \mathrm{MHz}$. They form the ridges which are characteristic for protons with strong dipolar hyperfine coupling, see Fig. 4c.

The HYSCORE spectra were analyzed using the method of Dikanov and Bowman [81]. This analysis yields for ${ }^{1} \mathrm{H}$ the parameters $\left|a_{\text {iso }}+T / 2\right|=3.5 \pm 0.1 \mathrm{MHz}$ and $|T|=5.9 \pm 0.1 \mathrm{MHz}$, for details see SI. Taking into account that for the electron-proton interaction the parallel principal value of the dipolar tensor is positive $(T$ $=+5.9 \pm 0.1 \mathrm{MHz}$ ), two possible isotropic hyperfine values, $a_{i s o}=+0.6 \pm 0.2 \mathrm{MHz}$ 


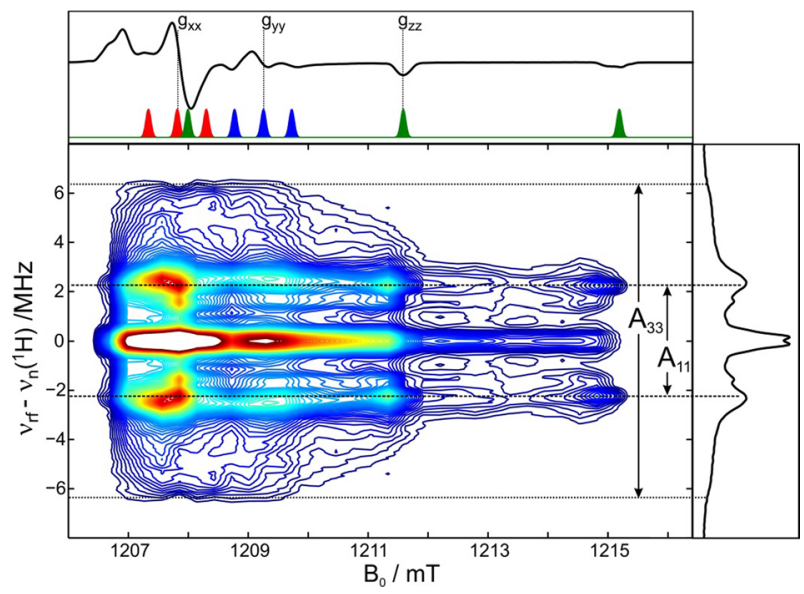

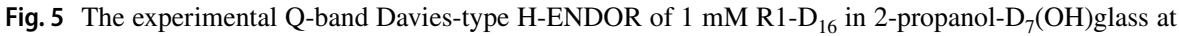
$60 \mathrm{~K}$ in contour plot representation. The contour lines are shown as isohypses from 0.05 to 1 of the maximum ENDOR signal intensity of the coupled proton. The trace on top shows the 2-pulse echo-detected Q-band EPR spectrum in first-derivative representation with the principal nitroxide g-tensor components with respective nitrogen hyperfine splittings indicated (in green, blue and red). The trace on the right side shows the powder-type ENDOR spectrum integrated over the field axis. The pulse settings of Daviestype ENDOR experiments are $t_{\pi}$ (pump) $=200 \mathrm{~ns}, \mathrm{t}_{\mathrm{RF}}=20 \mu \mathrm{s}, \mathrm{t}_{\pi / 2}($ det.) $=32 \mathrm{~ns}$; averages per point 160 , pulse repetition rate $500 \mathrm{~Hz}$, number of field points 40

and $a_{i s o}=-6.5 \pm 2 \mathrm{MHz}$, are derived from the above relations. A selection of the proper set of evaluated parameters cannot be based solely on the HYSCORE spectra. Thus, HYSCORE spectroscopy allows to obtain only the principal value of the dipolar hyperfine tensor, but delivers neither the proper value of the isotropic hyperfine coupling nor allows one to quantify possible non-axiality of a dipolar hyperfine tensor. Thus, the more direct ENDOR spectroscopy is required as a method to overcome the limitations of HYSCORE and to provide more precise information on the bonding situation.

\subsubsection{Multi-frequency ENDOR Experiments}

As next step, ENDOR experiments at X-band were performed. Unfortunately, in the center of the nitroxide EPR spectrum, the Davies ENDOR responses from bonded protons strongly overlap with intense signals caused by the $A_{x x}, A_{y y}{ }^{14} \mathrm{~N}$ hyperfine interaction (see Fig. S5b in SI). This makes the ENDOR traces difficult to analyze. Two well-resolved ENDOR lines positioned around the proton Larmor frequency can be detected at the low- and high-field spectral edges. They are determined by the high z-axis orientation selectivity of the $A_{z z}$ nitrogen hyperfine coupling (see Fig. S5 in SI). The proton hyperfine coupling of $4.55 \pm 0.10 \mathrm{MHz}$ is in good agreement with the value of $4.5 \mathrm{MHz}$ obtained from the analysis of the cw EPR line shape at the $g_{z z}, M_{I}=-1$ spectral position. 
In contrast to ENDOR at X-band, at Q-band microwave frequency, the proton and nitrogen hyperfine contributions are disentangled. The Davies-type H-ENDOR spectrum of $\mathrm{R} 1-\mathrm{D}_{16}$ in 2-propanol- $\mathrm{D}_{7}(\mathrm{OH})$ is shown in Fig. 5 in contour plot representation. The spectrum reveals several contributions. The minor contributions are (i) the intense matrix line at the proton Larmor frequency $\left(v_{n}\left({ }^{1} H\right)=51.5 \mathrm{MHz}\right.$ at $\left.1.21 \mathrm{~T}\right)$ which shows some structure due to coordinated 2-propanol molecules in the second nitroxide solvation sphere, and (ii) additional narrow lines below $\pm 2 \mathrm{MHz}$ around $v_{n}\left({ }^{1} H\right)$ due to unsubstituted internal nitroxide protons (see Sect. 14 in SI). The main contribution represents the typical dipolar pattern ("butterfly") of a dipolar coupled proton. It contains pronounced ridges at about $\pm 2 \mathrm{MHz}$ around $v_{n}\left({ }^{1} H\right)$ spread over the whole spectral range. An additional ridge of lower intensity with singularities at about $\pm 6.2 \mathrm{MHz}$ is formed in the $g_{x x}-g_{y y}$ spectral range, see Fig. 5. Both features are ascribed to perpendicular and parallel components of the proton dipolar hyperfine tensor, respectively. The perpendicular ridge is exclusively observed at the $g_{z z}$ field position between the middle-field $\left(M_{I}=0\right)$ and high-field $\left(M_{I}=-1\right)$ spectral regions. Thus, the hydrogen-bonded proton must lie in the $\mathrm{x}-\mathrm{y}$ plane of the nitroxide g-tensor axes system, i.e., the declination angle, $\Omega$, is very close to $90^{\circ}$, indicating a $\sigma$-type hydrogen bond, see Fig. 1b. A hyperfine coupling value $\left|A_{11}\right|=4.6 \pm 0.1 \mathrm{MHz}$ is obtained in $g_{z z}$ position. This agrees with the value derived from the cw EPR and X-band ENDOR spectra. A parallel dipolar coupling value of $\left|A_{33}\right|=12.4 \pm 0.1 \mathrm{MHz}$ is obtained from evaluation of the parallel ridges. At Q-band microwave frequency the nitroxide EPR spectrum is not fully resolved because $A_{z z} \approx \Delta g / g \cdot v_{0}$, see Fig. 5 top panel. Thus, neither the azimuthal angle, nor the $A_{22}$ hyperfine coupling value can be reliably obtained from the Q-band ENDOR spectrum.

The spectral resolution is significantly improved in W-band ENDOR. At W-band, the nitroxide EPR spectrum is completely disentangled in terms of the electron Zeeman interaction, i.e., the g-tensor. Figure 6a shows the W-band Davies H-ENDOR spectrum of the same sample solution used in the Q-band ENDOR experiment. The W-band spectra of the two nitroxide fractions become resolved at the $g_{x x}$ spectral position. Only ENDOR responses from the remote protons (summing up as "matrix line" at the proton Larmor frequency, $\left.v_{n}(H)\right)$ are detected at $g_{x x}(1)$, see Fig. 6a. This supports our conclusion drawn from the cw EPR results that the first nitroxide fraction has to be ascribed to free $\mathrm{R} 1$ radicals, i.e., without an H-bond to 2-propanol. In analogy to Q-band ENDOR, the pronounced perpendicular hyperfine ridges at about $\pm 2 \mathrm{MHz}$ around $v_{n}(H)$ are spread over the whole spectral range. The parallel ridge singularity at $\pm 6.2 \mathrm{MHz}$ appears at the well-defined parallel magnetic field position, $B_{||}$, between the $B_{x}$ and $B_{y}$ field values, see Fig. 6a, which correspond to the $g_{x x}$ and $g_{y y}$ canonical positions, respectively.

At this point, a few additional words regarding Zeeman orientation selection at high magnetic fields are appropriate: EPR at $B_{\|}$selects only those orientations of the nitroxides in frozen solution for which the field director is tilted by the azimuth angle away from the $\mathrm{x}$-axis in the $\mathrm{x}-\mathrm{y}$ plane of the radical, Fig. 1b. The orientation selection for the parallel dipolar coupling is given by the relation [68] (which acts as an orientation selector): 

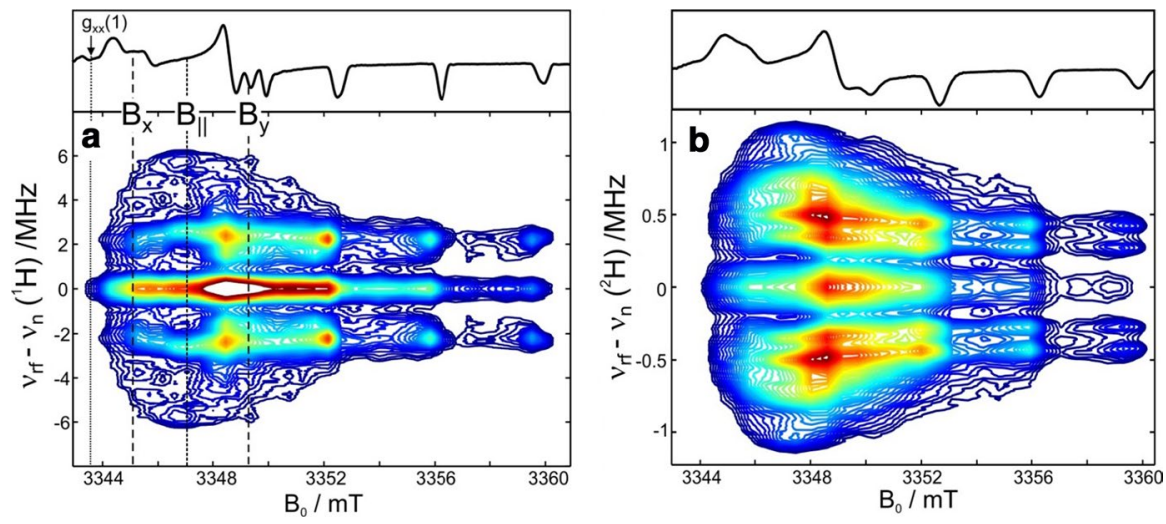

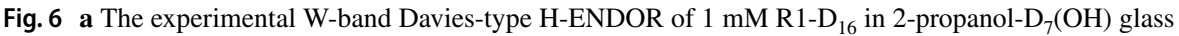
at $60 \mathrm{~K}$. The contour lines are shown as isohypses scaled from 0.05 to 1 of the maximum ENDOR intensity of the bonded proton. The trace on the top shows the 2-pulse echo-detected W-band EPR spectrum in first-derivative form. The pulse settings of Davies-type ENDOR experiments are $t_{\pi}$ (pump) $=200 \mathrm{~ns}$, $t_{R F}=20 \mu \mathrm{s}, t_{\pi / 2}$ (det.) $=32 \mathrm{~ns}$, averages per point 100 , pulse repetition rate $400 \mathrm{~Hz}$, number of field points 40 . The vertical dotted line marks the magnetic field position corresponding to $g_{x x}(1)$ of the radical fraction without hydrogen bond. b The experimental W-band Mims-type D-ENDOR of $1 \mathrm{mM} \mathrm{R} 1-\mathrm{H}_{16}$ in 2-propanol- $\mathrm{D}_{1}(\mathrm{OD})$ glass at $60 \mathrm{~K}$. The contour lines are shown as isohypses from 0.05 to 1 of the maximum ENDOR intensity. The trace on the top shows the 2-pulse echo-detected W-band EPR spectrum in first-derivative form. The pulse settings of Mims-type ENDOR experiments are $t_{\pi / 2}=32 \mathrm{~ns}, \mathrm{t}_{\mathrm{RF}}=30 \mu \mathrm{s}$, averages per point 100 , pulse repetition rate $400 \mathrm{~Hz}$, number of field points 40 . The spectrum is the average of two ENDOR spectra acquired with $\tau=250 \mathrm{~ns}$ and $400 \mathrm{~ns}$. Note, the RF frequency axis is scaled by the proton-to-deuterium nuclear g-value ratio of 6.51

$$
g(\theta, \varphi)^{2}=\left(g_{x x}^{2} \cdot \cos ^{2} \varphi+g_{y y}^{2} \cdot \sin ^{2} \varphi\right) \cdot \sin ^{2} \theta+g_{z z}^{2} \cdot \cos ^{2} \theta
$$

Thus, the parallel responses at the parallel frequency $\nu_{\|}$will show up at the parallel resonance-field position $B_{\| \mid}$within the nitroxide EPR spectrum where the electronnuclear dipolar vector coincides with the direction of the external magnetic field, i.e., $\theta=\Theta$ and $\varphi=\Phi$. Hence, the parallel hyperfine response is appearing at a unique parallel EPR resonance-field position

$$
B_{||}=\frac{h \cdot v_{0}}{g(\theta=\Theta, \varphi=\Phi) \cdot \mu_{B}}
$$

Taking into account that the hydrogen-bonded proton lies in the $\mathrm{x}-\mathrm{y}$ plane of the g-tensor frame, i.e., $\Theta=0$, Eq. (4) can be simplified to

$$
g_{x x}^{2} \cdot \cos ^{2} \Phi+g_{y y}^{2} \cdot \sin ^{2} \Phi=\left(\frac{\mu_{B} \cdot B_{\|}}{h \cdot v_{0}}\right)^{2}
$$

Using the EPR resonance condition for $g_{x x}$ and $g_{y y}$, i.e., $h \cdot v_{0}=g_{x x(y y)} \cdot \mu_{B} \cdot B_{x(y)}$, the azimuth angle can be calculated from the ratio of the resonance-field differences 
Fig. 7 Top and side views of the energy-optimized structure of the R1/2-propanol complex. The principal directions of the calculated nitroxide $\mathrm{g}$ - and bridged proton hyperfine tensors are indicated
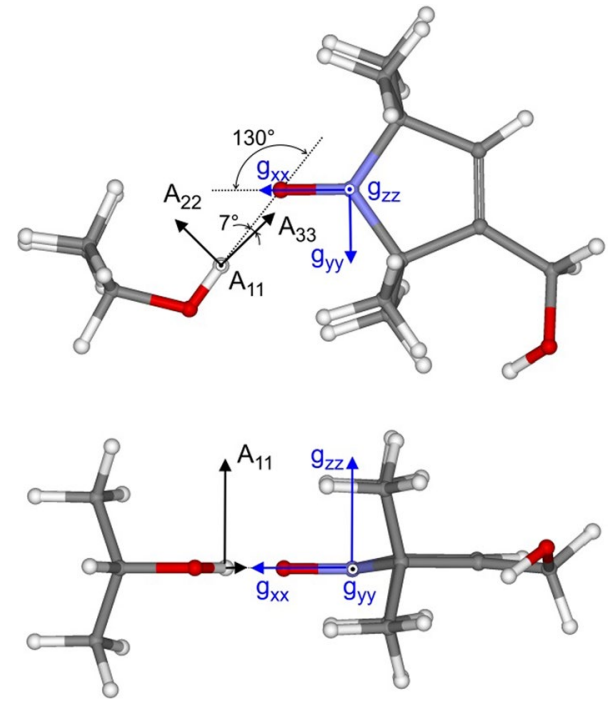

$$
\sin ^{2} \Phi=\frac{B_{\|}-B_{x}}{B_{y}-B_{x}}
$$

This ratio can easily be determined from the EPR spectrum even without knowledge of the absolute magnetic field values, provided linearity of the magnetic field sweep is secured. The field coordinate of the parallel hyperfine response is shifted by $B_{||}-B_{x}=1.9 \pm 0.2 \mathrm{mT}$ from the $g_{x x}$ resonance position. The difference between the $g_{x x}$ and $g_{y y}$ resonance fields, $B_{y}-B_{x}=4.0 \pm 0.1 \mathrm{mT}$, was determined from an echo-detected EPR spectrum that was recorded after the ENDOR experiment. It is in good agreement with the value $3.96 \mathrm{mT}$ calculated using the principal g-tensor components deduced from the cw EPR spectrum, i.e., $\left[g_{x x} ; g_{y y}\right]=[2.00843 ; 2.00606]$, and the actually used microwave frequency $v_{0}=93.99 \mathrm{GHz}$. Thus, from the resulting equation $\sin ^{2} \Phi=0.47 \pm 0.05$, the azimuth angle $\Phi=43 \pm 3^{\circ}$ (or $137 \pm 3^{\circ}$ ) can be calculated. Additionally, the missing ${ }^{1} \mathrm{H}$ hyperfine value of $\left|A_{22}\right|=5.5 \pm 0.1$ is evaluated from the ENDOR slice at the $B_{\|}$position because it coincides with the $A_{33}$ direction at $\Phi \approx 45^{\circ}$.

Thus, W-band ENDOR allows to obtain the complete ${ }^{1} \mathrm{H}$ hyperfine tensor of the hydrogen-bonded proton. Taking into account the positive sign of the nuclear g-value of the proton, $g_{n}>0$, also the signs of the hyperfine tensor principal values can be derived, i.e., $\left[A_{11}, A_{22}, A_{33}\right]=[-4.6,-5.5,+12.4] \mathrm{MHz}$. The direction of $A_{11}$ is collinear with the direction of $g_{z z}$, see Fig. 7. The $A_{33}$ director is rotated with respect to the $g_{x x}$ direction by $137^{\circ}$, using the $A_{i i}$ axes shown in Fig. 7.

Figure $6 \mathrm{~b}$ shows the Mims-type ENDOR spectrum of $\mathrm{R} 1-\mathrm{H}_{16}$ in 2-propanol$\mathrm{D}_{1}(\mathrm{OD})$, i.e., the ENDOR spectrum of R1 with hydrogen-bonded deuterium. The line shape reproduces that of the proton ENDOR spectrum of R1-D $\mathrm{D}_{16}$ in 2-propanol$\mathrm{D}_{7}(\mathrm{OH})$. However, all singularities are split due to the deuterium $(\mathrm{I}=1)$ quadrupole interaction. The analysis of the D-ENDOR spectrum allows one to determine the 
Table 2 Experimental and calculated (B3LYP/IGLO-III, COSMO) ${ }^{\text {a) }}$ magnetic parameters of the hydrogen-bridged proton (deuterium) in the R1/2-propanol hydrogen-bonded complex

\begin{tabular}{lll}
\hline Nuclei: Parameter & Experimental values & Calculated values \\
\hline $\mathrm{H}:\left[A_{11}, A_{22}, A_{33}\right] / \mathrm{MHz}$ & $-4.6,-5.5,+12.4 \pm 0.1^{\mathrm{b}}$ & $-4.54,-5.34,+11.08$ \\
$\mathrm{H}: a_{i s o} ; T / \mathrm{MHz} ; \eta$ & $+0.76 ;+5.82 ; 0.077$ & $+0.40 ;+5.34 ; 0.075$ \\
$\mathrm{D}:\left[A_{11}, A_{22}, A_{33}\right] / \mathrm{MHz}$ & $-0.70,-0.85,+1.90 \pm 0.01^{\mathrm{b}}$ & $-0.70,-0.82,+1.70$ \\
$\mathrm{D}:\left[P_{11}, P_{22}, P_{33}\right] / \mathrm{MHz}$ & $-0.053,-0.047,+0.100 \pm 0.003^{\mathrm{b})}$ & $-0.079,-0.057,+0.136$
\end{tabular}

${ }^{\text {a }}$ for the results of calculations using different basis sets, see SI

${ }^{\mathrm{b}}$ Experimental errors for hyperfine and quadrupole tensor components, see Fig S6 in SI

complete quadrupole tensor, $\tilde{P}$, and also to verify and refine the hyperfine tensor values, see Fig. S6 in SI. The results are summarized in Table 2. The simulations performed using the experimental values from both the deuterium and proton ENDOR spectra are in perfect agreement with the experimental recordings (see Figs. S7-9 in SI).

\subsubsection{DFT Results}

In this section, we compare the magnetic interaction parameters of the R1/2-propanol complex and unbound R1 with parameters obtained from state-of-the-art DFT calculations with the aim to support the analysis of our experimental observations.

4.1.4.1 H-bond Geometry Figure 7 shows the energy-optimized structure of R1 coordinated with 2-propanol (for atomic coordinates, see SI). The H-bonded bimolecular system represents a $\sigma$-type complex in which the hydrogen bond is formed with participation of the lone-pair electrons on the oxygen atom of the nitroxide radical. No difference was found for the two complex isomers with mirrored position of the 2-propanol molecule with respect to the hydroxymethyl group of the nitroxide. The calculated hydrogen bond lies in the plane of the nitroxide ring. The structure is characterized by a hydrogen bond length of $\mathrm{R}_{\mathrm{O} \cdots \mathrm{H}}=0.191 \mathrm{~nm}$, an almost linear $\mathrm{H}$-bond with $(\mathrm{OH} \cdots \mathrm{O})=178^{\circ}$. The hydrogen bond forms an angle of $(\mathrm{NO} \cdots \mathrm{H})=130^{\circ}$ which is close to the $120^{\circ}$ expected for the $\mathrm{sp}^{2}$ hybridized nitroxide oxygen [10]. The calculated H-bond geometries can be compared with the results of extensive DFT calculations on the $p$-benzosemiquinone radical anion coordinated with water molecules and various alcohols [25]. In general, the obtained H-bond angles are in good agreement with these calculations. The H-bond lengths are, however, much shorter for the radical anion than for the neutral radical due to the charge-dipole interplay. However, the H-bond length $(0.191 \mathrm{~nm})$ agrees well with that calculated for the neutral $p$-benzoquinone coordinated with a water molecule $(0.195 \mathrm{~nm})$ [25] and for different nitroxides coordinated with alcohols or water, for which the values range from $0.177 \mathrm{~nm}$ to $0.194 \mathrm{~nm}[59-61,82,83]$.

4.1.4.2 Magnetic Parameters of the Bridging Proton (Deuterium) The calculated principal directions of the bridged proton hyperfine tensor are shown in Fig. 7 overlaid with the molecular structure of the nitroxide complex together with its g-tensor axes. The calculated $A_{11}$ direction is perfectly collinear with the $g_{z z}$-direction and both 
Table 3 Experimental and calculated (B3LYP/IGLO-III, COSMO)* magnetic parameters of nitroxide $\mathrm{R} 1$ in the unbound form and the R1/2-propanol complex

\begin{tabular}{|c|c|c|c|}
\hline & Parameter & Experimental values & Calculated values \\
\hline \multirow[t]{4}{*}{$\mathrm{R} 1 \cdots \mathrm{HO}$} & {$\left[g_{x x}, g_{y y}, g_{z z}\right]-2 / 10^{-6}$} & $8432,6062,2185$ & $8453,6050,2155$ \\
\hline & $g_{\text {iso }}-2 / 10^{-6}$ & 5560 & 5553 \\
\hline & ${ }^{14} \mathrm{~N}: a_{i s o} ; T / \mathrm{MHz}$ & $42.07 ; 28.80$ & $29.60 ; 29.20$ \\
\hline & ${ }^{14} \mathrm{~N}: e^{2} q \cdot h^{-1} Q / \mathrm{MHz} ; \eta$ & $-3.240 ; 0.52$ & $-3.852 ; 0.57$ \\
\hline \multirow[t]{4}{*}{$\mathrm{R} 1$} & {$\left[g_{x x}, g_{y y}, g_{z z}\right]-2 / 10^{-6}$} & $9109,6196,2185$ & $8900,6248,2118$ \\
\hline & $g_{\text {iso }}-2 / 10^{-6}$ & 5830 & 5755 \\
\hline & ${ }^{14} \mathrm{~N}: a_{i s o} ; T / \mathrm{MHz}$ & $39.10 ; 28.20$ & $28.3 ; 28.20$ \\
\hline & ${ }^{14} \mathrm{~N}: e^{2} q \cdot h^{-1} Q / \mathrm{MHz} ; \eta$ & $-3.54 ; 0.43$ & $-3.96 ; 0.49$ \\
\hline
\end{tabular}

*For the results of calculation using different basis sets and for all principal values, see SI

directions are perpendicular to the $\mathrm{OH} \cdots \mathrm{ON}$ plane. The angle between the hyperfine 3 -axis and $g_{x x}$-axis is $137.7^{\circ}$ with the hyperfine $\mathrm{x}$-axis rotated toward the nitroxide nitrogen which carries substantial spin density. This angle is in very good agreement with the angle of $(137 \pm 3)^{\circ}$ obtained experimentally. Thus, we conclude that the real molecular structure of the R1/2-propanol complex should be very similar to that depicted in Fig. 7. The calculated principal values of the hyperfine and quadrupole tensors of the bridging proton (deuterium) for R1 coordinated with 2-propanol are summarized in Table 2 (for details see SI). The experimental asymmetry (rhombicity) parameter of the dipolar hyperfine tensor, $\eta=0.077 \pm 0.001$, is well reproduced by the calculations, $\eta=0.075$. The calculated principal values of the ${ }^{1} \mathrm{H}$ hyperfine tensor are somewhat smaller than the experimental ones; thus, the isotropic coupling is underestimated. The calculated principal directions of the bridge-deuterium quadrupole tensor coincide within $6^{\circ}$ with the corresponding hyperfine tensor directions (for details, see SI), in agreement with experiment. The respective quadrupole tensor values for the deuterium in the bridge are, however, somewhat overestimated by the calculation leading to a rather poor agreement of the nuclear quadrupole coupling and asymmetry parameter values.

4.1.4.3 Nitroxide Radical Magnetic Parameters The calculated and experimental magnetic parameters of the nitroxide for the R1/2-propanol complex and the unbound $\mathrm{R} 1$ radical are summarized in Table 3 . The calculated principal directions of the nitrogen hyperfine and quadrupole tensors are collinear with the corresponding directions of the $g$-tensor agreeing within $2^{\circ}$; this supports our previous experimental findings [63]. As expected, the isotropic g-factor, $g_{i s o}$, decreases upon formation of the complex. This is caused by a shift of spin density from $\mathrm{O}$ to $\mathrm{N}$ in the nitroxide upon formation of the hydrogen bond (note, oxygen has a larger spin orbit coupling than nitrogen). The calculated $g_{\text {iso }}$ difference of $2.0 \cdot 10^{-5}$, however, is somewhat smaller than the experimental difference of $2.7 \cdot 10^{-5}$. The calculation yields an axially symmetric dipolar ${ }^{14} \mathrm{~N}$ hyperfine tensor in agreement with experiment. The experimental and calculated principal dipolar ${ }^{14} \mathrm{~N}$ hyperfine tensor values, $T$, agree to first approximation. The increase of $T$ by $0.6 \mathrm{MHz}$ (experiment) and $1.0 \mathrm{MHz}$ (calculation) is 
consistent with the increase of electron spin density at the ${ }^{14} \mathrm{~N}$ upon formation of the $\mathrm{H}$-bond and consistent with the increase of the isotropic ${ }^{14} \mathrm{~N}$ hyperfine coupling, $a_{i s o}$, by $3 \mathrm{MHz}$ (experiment) and $1.3 \mathrm{MHz}$ (calculation). Both the difference and the $a_{\text {iso }}$ values are underestimated in the calculation. In contrast, the magnitude of the principal quadrupole values, $\left|e^{2} q \cdot h^{-1} Q\right|$, are overestimated, and the difference is underestimated. The calculated quadrupole asymmetry parameter and its change upon complex formation agree with the experimental values. In general, the calculation results are in agreement with the results of our previous investigations [70]. However, the lack of accuracy in the calculations indicates that a larger model system with inclusion of the solvent shell is necessary to obtain a better agreement between calculation and experiment (e.g., attempts in ref. [25], on benzoquinone). There is also the possibility that the hydroxymethyl group of the nitroxide is involved in additional interactions with the solvent matrix (e.g., via H-bonding) changing the geometry of the bimolecular complex. This has so far not been taken into account.

\subsection{Nitroxide R2, the Six-Membered Ring System}

Besides some small disagreements between the interaction-parameter values obtained by DFT calculations and experiments, the $\sigma$-type structure of the R1/2propanol complex, as shown in Fig. 7, is considered a solid result. This conclusion, however, is in contradiction to several previous reports on nitroxide radicals in the literature. For example, liquid-solution NMR studies of nitroxide radicals dissolved in alcohols revealed proton hyperfine couplings interpreted with a $\pi$-type structure of the complex $[66,67]$. Also, the recent W-band ENDOR study of the nitrosodisulfonate anion (Fremy salt) in binary methanol/water solution favored a $\pi$-type complex [84]. On the other hand, a $\sigma$-type complex has been derived from D-band $(130 \mathrm{GHz})$ ENDOR experiments of a protonated nitroxide radical (the lipid bilayer spin probe 5-doxyl stearic acid) in different OD-labeled alcohols [85]. Notably, the reported D-band $(130 \mathrm{GHz})$ ENDOR spectra did not resolve a deuterium quadrupole splitting which, however, is clearly resolved in the W-band ENDOR spectrum of the R1/2-propanol complex, Figs. $6 \mathrm{~b}$ and S6 in SI. Moreover, the hyperfine parameters of the bridged deuterium derived from the D-band ENDOR spectra simulation [85], $\left[A_{11}, A_{22}, A_{33}\right]=[0.72,1.60,-1.76] \mathrm{MHz}$, strongly disagree with the values obtained for the R1/2-propanol complex, see Table 2.

An obvious explanation for such discrepancies could be a dependence of the geometry of the radical-solvent complex on the molecular structure of the nitroxide radical. In contrast to the $\mathrm{R} 1$ nitroxide, characterized by the near planarity of the five-membered ring with a negligibly small bending angle of the NO bond with respect to the ring plane, the radicals employed so far in NMR and EPR studies have non-planar structures with a larger $\mathrm{NO}$ bond angle with respect to the $\mathrm{C}-\mathrm{N}-\mathrm{C}$ (or S-N-S for nitrosodisulfonate anion) plane. To check this hypothetical explanation, we performed a W-band ENDOR study of the radical R2 in 2-propanol. The $\mathrm{R} 2$ radical is one of the typical nitroxides frequently employed in previous EPR and NMR investigations. The chair conformation of the six-membered ring radical R2 has an out-of-plane bending angle of $19^{\circ}$ with respect to the adjacent $\mathrm{C}-\mathrm{N}-\mathrm{C}$ plane. 


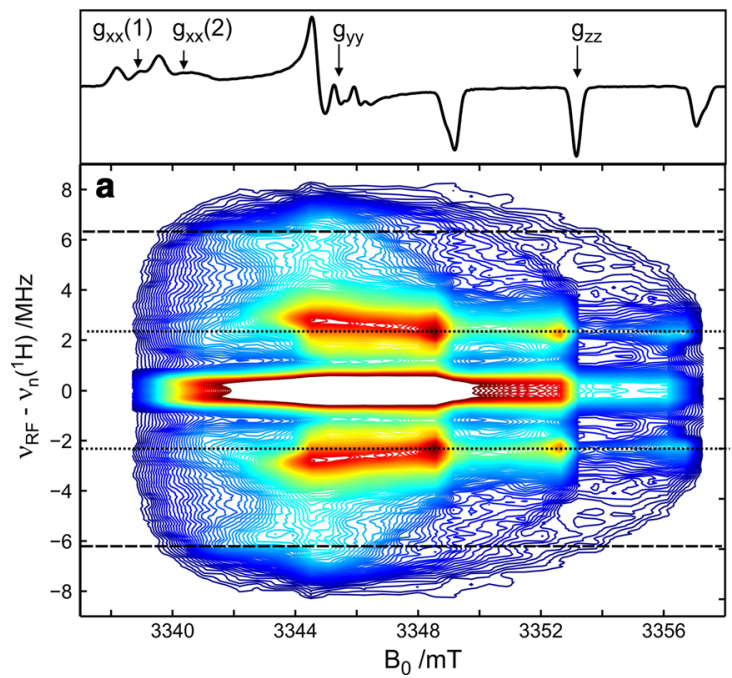

b
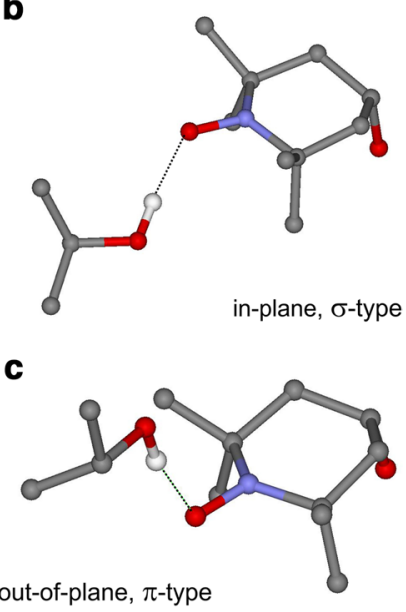

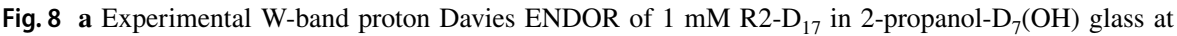
$60 \mathrm{~K}$. The contour lines are shown as isohypses from 0.05 to 1 of the maximum ENDOR intensity of the coupled proton contribution. The horizontal dotted and dashed lines mark the frequency positions of parallel and perpendicular hyperfine responses of the bridged proton in a $\sigma$-type complex The trace on the top shows the 2-pulse echo-detected W-band EPR spectrum in first-derivative form. The pulse settings of Davies-type ENDOR experiments are $\mathrm{t}_{\pi}($ pump $)=200 \mathrm{~ns}, \mathrm{t}_{\mathrm{RF}}=20 \mu \mathrm{s}, \mathrm{t}_{\pi / 2}($ det. $)=32 \mathrm{~ns}$, averages per point 160 , pulse repetition rate $500 \mathrm{~Hz}$, number of field points 40 . (b, c) Energy optimized structures of $\sigma$ - and $\pi$-types of the R2/2-propanol complex. The protons, except for the bridged one, are omitted for clarity. For details, see text

Our previous W-band cw EPR and ELDOR-detected NMR investigations of R2 in 2-propanol showed a similar situation as for R1 in 2-propanol [63]. Two different nitroxide subensembles in the EPR and EDNMR spectra were clearly distinguished. $\mathrm{R} 2$ reveals a slightly smaller fraction ( 0.5 vs. 0.6 for $\mathrm{R} 1)$ of radicals with a lower $g_{x x}$ (higher $A_{z z}$ ) value. Figure 8 a shows the Davies ENDOR spectrum of $1 \mathrm{mM}$ R2-D 17 in 2-propanol- $\mathrm{D}_{7}(\mathrm{OH})$ frozen solution recorded at $60 \mathrm{~K}$. Analogous to R1-D 16 in 2-propanol- $\mathrm{D}_{7}(\mathrm{OH})$, ENDOR responses of strongly coupled protons appear only at the field position corresponding to the radical fraction with the lower $g_{x x}$-value. Perpendicular ridges, at the same frequency positions as in the R1 case, are observed across the EPR spectral range, see dotted horizontal lines in Fig. 8a. The distribution of ENDOR intensities of R2/2-propanol at higher frequencies differs substantially from that of the R1/2-propanol ENDOR, Fig. S10 in SI. The ENDOR intensities are spread over the whole EPR spectral range. However, the singularities at $\pm 6.4 \mathrm{MHz}$ can be recognized at the same spectral position between $g_{x x}$ and $g_{y y}$, see dashed horizontal lines in Fig. 8a and Fig S10 in SI. Additional singularities at about $\pm 7.6 \mathrm{MHz}$ are shifted toward the $g_{z z}$ spectral position. The last singularities are ascribed to parallel responses of the R2/2-propanol complex which has a different geometry compared to the R1 case. The corresponding perpendicular responses are not observed.

Thus, the R2/2-propanol ENDOR spectrum is interpreted as a mixture of at least two proton ENDOR spectra of complexes with different geometry. The first 
contribution to the R2/2-propanol ENDOR spectrum with principal proton hyperfine coupling values $\left[A_{11}, A_{22}, A_{33}\right]=[-4.6,-5.9,12.8] \pm 0.2 \mathrm{MHz}$ and the azimuth angle $\Phi=135 \pm 5^{\circ}$, which is very close to the values of the R1/2-propanol complex, is ascribed to a $\sigma$-type complex.

For the second contribution to the R2/2-propanol ENDOR spectrum in Fig. 8a, the magnetic interaction parameters of this complex cannot be reliably extracted from the ENDOR responses. Hence, for R2/2-propanol, additional DFT calculations were performed at the same level as for the R1/2-propanol case (see Sect. 4.1.4). The energy optimization of the complex always resulted in a $\sigma$-type structure with an almost linear $\mathrm{H}$-bond with $(\mathrm{OH} \ldots \mathrm{O}) \approx 175^{\circ},(\mathrm{NO} \ldots \mathrm{H}) \approx 133^{\circ}$ and a hydrogen bond length of $\mathrm{R}_{\mathrm{O} \ldots \mathrm{H}}=0.191 \mathrm{~nm}$, see Fig. 8b. However, four conformers with respect to the nitroxide hydroxyl group and to the nitroxide chair plane are very close in energy (within $\pm 0.4 \mathrm{~kJ} / \mathrm{mol}$ ). The calculated principal hyperfine values of the bridged proton are slightly different for different conformers, having the hyperfine tensor components of $\left[A_{11}, A_{22}, A_{33}\right]=[-6.1,-6.8,+11.8] \mathrm{MHz}$ within $\pm 0.3 \mathrm{MHz}$, and are close to those of the R1/2-propanol complex, see Table 2. The calculated principal hyperfine directions with respect to the nitroxide g-tensor frame differ also slightly for all conformers. The calculated azimuth angle falls, however, in the error range of experimental value of $\Phi=135 \pm 5^{\circ}$.

The calculated ENDOR spectrum using DFT hyperfine tensors agrees well with the experimental ENDOR spectrum when considering the contributions of the $\sigma$-type R2/2-propanol fraction, see Fig. S12a in the SI. We note that the experimental ENDOR responses are much broader for R2/2-propanol than for R1/2-propanol, which becomes obvious from a direct comparison of the ENDOR spectra, see Fig. S11 in SI. Presumably, this broadening is caused by the larger number of conformers of the R2/2-propanol complex and/or a larger matrix effect of bulk solvent molecules on the non-planar R2 structure that results in a distribution of hyperfine coupling values and directions.

To calculate the probability of other types of structures of R2/solvent complexes, the starting geometry was selected to be close to $\pi$-type, and the total energy optimization was restricted to a range of angles $\pm 30^{\circ}$ around the initial position, i.e., the proton directly over the $\mathrm{p}_{\mathrm{z}}$ orbital of the oxygen atom. As a result, the energies of the calculated structures converge reproducibly to the local energy minimum close to that of a $\pi$-type structure with a hydrogen bond length of $\mathrm{R}_{\mathrm{O} \cdots \mathrm{H}}=0.191 \mathrm{~nm}$, $(\mathrm{OH} \cdots \mathrm{O}) \approx 170^{\circ},(\mathrm{NO} \cdots \mathrm{H}) \approx 111^{\circ}$ and $\approx 70^{\circ}$ out of the oxygen $\sigma$-orbital plane, see Fig. 8c. The energy of the $\pi(\mathrm{O})$-type complex is, however, about $14 \mathrm{~kJ} / \mathrm{mol}$ higher then that of the $\sigma$-complex. Thus, a very low population of the $\pi(\mathrm{O})$-type complex is expected $(0.3 \%$ at $298 \mathrm{~K})$. The calculated principal hyperfine tensor values of $\left[A_{11}\right.$, $\left.A_{22}, A_{33}\right]=[-13.60,-12.29,+14.34] \mathrm{MHz}$ show significant negative electron spin density on the bridged proton, $a_{i s o}=-3.85 \mathrm{MHz}$. The calculated values agrees well with the result of ab initio calculations for $\pi$-type R2/water complex ([ $\left.A_{11}, A_{22}, A_{33}\right]$ $=[-12.60,-11.8,+14.2] \mathrm{MHz}, a_{i s o}=-3.38 \mathrm{MHz}$ ) [86]. Moreover, these values are close to $[-14.6,-14.3,+15.1] \mathrm{MHz}$ obtained for $\mathrm{R}_{2}-\mathrm{D}_{17}$ in the single crystal diamagnetic host (4-hydroxy-2,2,6,6-tertamethylpiperidine) from the analysis of Q-band cw EPR spectra [87]. The larger $A_{33}$-value agrees also with the frequency position of the second parallel singularity observed in our experimental ENDOR 
spectrum. However, the linear combination of ENDOR spectra using DFT hyperfine tensors of both complex types does not fully reproduce the experimental spectrum (see Fig. S12 in SI). This is surprising because from the comparison of experimental and calculated ENDOR intensities the two fractions of complexes should be of comparable magnitude. Thus, the formation of the $\pi$-type complex looks to be dubious considering the above DFT results. We note, however, that the calculated structure was optimized in vacuo. Bulk solvent molecules may affect the structure of the nitroxide itself, and as result the structure of the R2/solvent complex and the energy gap between the $\sigma$ and $\pi$ complex types may be changed.

The ENDOR and DFT data thus obtained show substantial differences between the R1/2-propanol and R2/2-propanol complexes. The structure of the unique $\sigma$-type $\mathrm{R} 1 / 2$-propanol is well defined mostly because of the planarity of the nitroxide ring and the minor influence of other solvent (bulk) molecules on the geometry of the complex. The R2/2-propanol complex exists in at least two conformations of comparable fractions, both with less defined geometry than the $\sigma$-type complex of R 1 in 2-propanol. This might explain the discrepancy of the interpretations of high-field ENDOR results published in the past on this system [84, 85].

As a final note, we want to compare the measurements performed at room temperature using NMR with the EPR/ENDOR results performed on dilute frozen solutions. Earlier evaluations of alcohol hydroxyl proton paramagnetic shifts induced by formation of H-bond complexes with piperidine-type nitroxides (like R2) yielded a negative proton isotropic hyperfine constant of about $a_{i s o}=-1.2 \mathrm{MHz}$ at RT $[30,35]$. This observation is best explained by the formation of a $\pi$-type complex in which the proton hfc is rather large and negative. In case of a $\sigma$-type (in-plane) complex a very small positive hfc is obtained that changes sign only for very short H-bonds $(<0.165 \mathrm{~nm})$ [25].

The proton isotropic hyperfine constant of $-1.2 \mathrm{MHz}$ reported by NMR is much smaller than the value of $-3.85 \mathrm{MHz}$ we have calculated for the $\pi$-type $\mathrm{R} 2 / 2$ propanol complex by DFT and smaller than other values calculated previously by ab initio methods [86]. To shed light on this problem, we performed additional NMR measurements in liquid solution detailed in the SI. For both radicals, the positive NMR contact shift obtained for the hydroxyl proton of 2-propanol indicates a negative isotropic proton hyperfine coupling. However, the $\left|a_{i s o}\right|$ value estimated from the NMR contact shifts in R1/2-propanol is about a factor of 4.5 smaller than that of R2/2-propanol (see SI). Furthermore, the negative sign of $a_{i s o}$ in R1/2-propanol disagrees with the positive proton isotropic coupling in the $\sigma$-type R1/2-propanol complexes detected by EPR/ENDOR in frozen solution. This problem could, however, be solved by assuming a dynamic formation of both $\sigma$ and $\pi$ complexes with different equilibrium constants in the liquid solution at room temperature. If only formation of $\sigma$ and $\pi$ complexes is assumed for the R 1 radical, the equilibrium has to be shifted toward the $\sigma$-complex formation to compensate the large negative contact shift due to the $\pi$-complex formation by the small positive value of the $\sigma$-complex. Thus, both radicals R1 and R2 have to form both $\sigma$-type and $\pi$-type short-lived dynamic complexes in liquid solution at RT. In frozen solution, however, solvent dynamics and internal motion of the nitroxide radical, for instance, out-ofplane motion of the nitroxide oxygen [80], are prohibited or largely restricted. As a 
result, upon slow (in comparison with the lifetime of the complex in liquid solution) cooling the $\sigma$-type R1/2-propanol complex is stabilized and exclusively detected by ENDOR at low temperatures.

It is important to note, that the results of our NMR experiments are in good agreement with findings of previous liquid-solution NMR studies [88] that showed significantly smaller but still negative $a_{i s o}$ values for a planar 6-membered ring nitroxide (2-oxo-TEMPO) complexed with ionol as compared to the nonplanar R2.

\section{Conclusion}

Multi-frequency multi-resonance EPR and ENDOR investigations of two structurally different nitroxide radicals hydrogen-bonded with 2-propanol are presented. The planar five-membered ring system of a pyrroline-type nitroxide radical, R1, was found to form exclusively a well-defined in-plane $\sigma$-type hydrogen-bonded complex with a solvent molecule in frozen 2-propanol solution. The experimental hyperfine parameters of the H-bridge proton and of the internal magnetic interaction parameters (g-, hyperfine-, quadrupole-tensor components) of R1 are in good agreement with values predicted by state-of-the-art DFT calculations. In contrast, the non-planar six-membered ring system of a piperidine-type nitroxide radical, R2, was found to form a mixture of less well-defined $\sigma$-type with presumably $\pi$-type complexes, both types of complexes contributing with comparable fractions. The DFT calculations of the magnetic interaction parameters show satisfying agreement with the experiments for nitroxide R1 interacting with 2-propanol but failed to predict trustable parameters for R2, probably due to the substantial flexibility of the nitroxide and its hydrogen-bonded complexes. Obviously, this failure is due to the limited model used in the calculations, which did not include the impact of the second solvation shell.

We conclude that high-field ENDOR in conjunction with DFT calculation is the method of choice for the investigation of hydrogen bonding of nitroxide radicals. We believe that the new information about hydrogen bonding of nitroxide radicals will allow to improve the prediction methods for investigation of nitroxide-labeled (bio)-macromolecules (DEER rotamer library [89]) and also lead to a better understanding of the dependencies of Overhauser DNP [90] enhancement factors on the structure of nitroxides [91].

Supplementary Information The online version contains supplementary material available at https://doi. org/10.1007/s00723-021-01442-y.

Acknowledgements We thank Yuri Grishin (Institute of Chemical Kinetics and Combustion, Novosibirsk, Russia) for enduring technical support and Herbert Zimmermann (Max-Planck Institute for Medical Research, Heidelberg) for providing the isotope labelled forms of the nitroxide radical R1. We thank Gudrun Klihm (Max Planck Institute for Chemical Energy Conversion) for assistance with the $244 \mathrm{GHz}$ EPR spectrometer. This work is supported by the Deutsche Forschungsgemeinschaft and the Max-PlanckGesellschaft. K.M. and M.P. acknowledge sustaining support by Freie Universität Berlin.

Funding Open Access funding enabled and organized by Projekt DEAL. 
Open Access This article is licensed under a Creative Commons Attribution 4.0 International License, which permits use, sharing, adaptation, distribution and reproduction in any medium or format, as long as you give appropriate credit to the original author(s) and the source, provide a link to the Creative Commons licence, and indicate if changes were made. The images or other third party material in this article are included in the article's Creative Commons licence, unless indicated otherwise in a credit line to the material. If material is not included in the article's Creative Commons licence and your intended use is not permitted by statutory regulation or exceeds the permitted use, you will need to obtain permission directly from the copyright holder. To view a copy of this licence, visit http://creativecommons.org/licen ses/by/4.0/.

\section{References}

1. E. Arunan, G.R. Desiraju, R.A. Klein, J. Sadlej, S. Scheiner, I. Alkorta, D.C. Clary, R.H. Crabtree, J.J. Dannenberg, P. Hobza, H.G. Kjaergaard, A.C. Legon, B. Mennucci, D.J. Nesbitt, Pure Appl. Chem. 83, 1637-1641 (2011)

2. G.R. Desiraju, T. Steiner, The Weak Hydrogen Bond in Structural Chemistry and Biology (Oxford University Press, 1999)

3. G. Gilli, P. Gilli, The Nature of the Hydrogen Bond (Oxford University Press, 2009)

4. S.J. Grabowski (ed.), Hydrogen Bonding-New Insights (Springer, 2006)

5. G.A. Jeffrey, An Introduction to Hydrogen Bonding (Oxford University Press, 1997)

6. G.A. Jeffrey, W. Saenger, Hydrogen Bonding in Biological Structures (Springer, 1991)

7. P.M. Pihko (ed.), Hydrogen Bonding in Organic Synthesis (Wiley-VCH, 2009)

8. L. Pauling, The Nature of the Chemical Bond, and the Structure of Molecules and Crystals: An Introduction to Modern Structural Chemistry (Cornell University Press Ithaca, 1960)

9. T.S. Moore, T.F. Winmill, J. Chem. Soc., Trans. 101, 1635-1676 (1912)

10. T. Steiner, Angew. Chem. Int. Ed. 41, 48-76 (2002)

11. W. Lubitz, G. Feher, Appl. Magn. Reson. 17, 1 (1999)

12. M. Flores, R. Isaacson, E. Abresch, R. Calvo, W. Lubitz, G. Feher, Biophys. J. 92, 671-682 (2007)

13. M. Flores, R. Isaacson, E. Abresch, R. Calvo, W. Lubitz, G. Feher, Biophys. J. 90, 3356-3362 (2006)

14. M. Flores, A. Savitsky, M.L. Paddock, E.C. Abresch, A.A. Dubinskii, M.Y. Okamura, W. Lubitz, K. Möbius, J. Phys. Chem. B. 114, 16894-16901 (2010)

15. A.T. Gardiner, S.G. Zech, F. MacMillan, H. Käss, R. Bittl, E. Schlodder, F. Lendzian, W. Lubitz, Biochem. 38, 11773-11787 (1999)

16. E. Martin, R.I. Samoilova, K.V. Narasimhulu, T.-J. Lin, P.J. O’Malley, C.A. Wraight, S.A. Dikanov, J. Am. Chem. Soc. 133, 5525-5537 (2011)

17. A.T. Taguchi, P.J. O’Malley, C.A. Wraight, S.A. Dikanov, J. Phys. Chem. B. 118, 9225-9237 (2014)

18. C. Teutloff, R. Bittl, W. Lubitz, Appl. Magn. Reson. 26, 5 (2004)

19. J. Niklas, B. Epel, M.L. Antonkine, S. Sinnecker, M.-E. Pandelia, W. Lubitz, J. Phys. Chem. B. 113, 10367-10379 (2009)

20. A. van der Est, Y. Pushkar, I. Karyagina, B. Fonovic, T. Dudding, J. Niklas, W. Lubitz, J.H. Golbeck, Appl. Magn. Reson. 37, 65 (2009)

21. Y.N. Pushkar, D. Stehlik, M. van Gastel, W. Lubitz, J. Mol. Struct. 700, 233-241 (2004)

22. K. Saito, A.W. Rutherford, H. Ishikita, Proc. Natl. Acad. Sci. USA 110, 954 (2013)

23. B. Epel, J. Niklas, S. Sinnecker, H. Zimmermann, W. Lubitz, J. Phys. Chem. B. 110, 11549-11560 (2006)

24. F. MacMillan, F. Lendzian, W. Lubitz, Magn. Reson. Chem. 33, S81-S93 (1995)

25. S. Sinnecker, E. Reijerse, F. Neese, W. Lubitz, J. Am. Chem. Soc. 126, 3280-3290 (2004)

26. M. Flores, R.A. Isaacson, R. Calvo, G. Feher, W. Lubitz, Chem. Phys. 294, 401-413 (2003)

27. G.I. Likhtenshtein, J. Yamauchi, S.I. Nakatsuji, A.I. Smirnov, R. Tamura, Nitroxides. Applications in Chemistry, Biomedicine, and Materials Science (Wiley-VCH, 2008)

28. L.J. Berliner, Biological Magnetic Resonance Vol. 14, Spin Labeling (The nest Millennium, 2002)

29. E. Dally, W. Müller-Warmuth, Ber. Bunsenges. Phys. Chem. 81, 1133-1137 (1977)

30. E. Dally, W. Müller-Warmuth, Ber. Bunsenges. Phys. Chem. 82, 792-798 (1978) 
31. E. Dally, W. Müller-Warmuth, Ber. Bunsenges. Phys. Chem. 84, 260-265 (1980)

32. K. Endo, Y. Hazama, K. Okabayashi, I. Tonoike, K. Suzuki, Chem. Phys. Lett. 61, 336-338 (1979)

33. K. Endo, I. Morishima, T. Yonezawa, J. Chem. Phys. 67, 4760-4767 (1977)

34. W. Kolodziejski, Z. Kecki, J. Magn. Reson. 28, 63-67 (1977)

35. W. Kolodziejski, Z. Kecki, Ber. Bunsenges. Phys. Chem. 82, 1312-1314 (1978)

36. I. Morishima, K. Endo, T. Yonezawa, J. Chem. Phys. 58, 3146-3154 (1973)

37. I. Morishima, K. Ishihara, K. Tomishima, T. Inubushi, T. Yonezawa, J. Am. Chem. Soc. 97, 27492756 (1975)

38. L. Morishima, K. Endo, T. Yonezawa, Chem. Phys. Lett. 9, 143-146 (1971)

39. N.A. Sysoeva, A.Y. Karmilov, A.L. Buchachenko, Chem. Phys. 7, 123-129 (1975)

40. N.A. Sysoeva, A.Y. Karmilov, A.L. Buchachenko, Chem. Phys. 15, 321-330 (1976)

41. P. Franchi, M. Lucarini, P. Pedrielli, G.F. Pedulli, ChemPhysChem 3, 789-793 (2002)

42. Y.Y. Lim, R.S. Drago, J. Am. Chem. Soc. 93, 891 (1971)

43. B. Chion, J. Lajzerowicz-Bonneteau, Acta Cryst. B. 36, 998-1000 (1980)

44. S. Nakajima, E. Kato, M. Minatozaki, H. Nishide, A supramolecular polymer of nitroxide radicals via hydrogen bonding, in Macromolecular complexes. ed. by J. Costamagna, F.M. Rabagliati, B.L. Rivas (Springer, 2011), pp. 1-7

45. B. Ahrens, M.G. Davidson, V.T. Forsyth, M.F. Mahon, A.L. Johnson, S.A. Mason, R.D. Price, P.R. Raithby, J. Am. Chem. Soc. 123, 9164-9165 (2001)

46. L.R. Mahoney, G.D. Mendenhall, K.U. Ingold, J. Am. Chem. Soc. 95, 8610-8614 (1973)

47. A.L. Buchachenko, O.P. Sukhanova, J. Struct. Chem. 6, 24-29 (1965)

48. T. Kawamura, S. Matsunam, T. Yonezawa, Bull. Chem. Soc. Jpn. 40, 1111 (1967)

49. O.H. Griffith, P.J. Dehlinger, S.P. Van, J. Membr. Biol. 15, 159-192 (1974)

50. H.J. Steinhoff, A. Savitsky, C. Wegener, M. Pfeiffer, M. Plato, K. Möbius, Biochim. Biophys. Acta. 1457, 253-262 (2000)

51. L. Urban, H.-J. Steinhoff, Mol. Phys. 111, 2873-2881 (2013)

52. E. Bordignon, H. Brutlach, L. Urban, K. Hideg, A. Savitsky, A. Schnegg, P. Gast, M. Engelhard, E.J.J. Groenen, K. Möbius, H.J. Steinhoff, Appl. Magn. Reson. 37, 391-403 (2010)

53. E. Bordignon, A.I. Nalepa, A. Savitsky, L. Braun, G. Jeschke, J. Phys. Chem. B. 119, 13797-13806 (2015)

54. A. Nalepa, K. Möbius, M. Plato, W. Lubitz, A. Savitsky, Appl. Magn. Reson. 50, 1-16 (2019)

55. T.I. Smirnova, T.G. Chadwick, M.A. Voinov, O. Poluektov, J. van Tol, A. Ozarowski, G. Schaaf, M.M. Ryan, V.A. Bankaitis, Biophys. J. 92, 3686-3695 (2007)

56. P. Gast, R.T.L. Herbonnet, J. Klare, A. Nalepa, C. Rickert, D. Stellinga, L. Urban, K. Möbius, A. Savitsky, H.J. Steinhoff, E.J.J. Groenen, Phys. Chem. Chem. Phys. 16, 15910-15916 (2014)

57. R. Owenius, M. Engström, M. Lindgren, M. Huber, J. Phys. Chem. A. 105, 10967-10977 (2001)

58. Z. Rinkevicius, N.A. Murugan, J. Kongsted, K. Aidas, A.H. Steindal, H. Agren, J. Phys. Chem. B. 115, 4350-4358 (2011)

59. Z. Rinkevicius, L. Telyatnyk, O. Vahtras, K. Ruud, J. Chem. Phys. 121, 5051-5060 (2004)

60. M. Pavone, P. Cimino, O. Crescenzi, A. Sillanpaeae, V. Barone, J. Phys. Chem. B. 111, 8928-8939 (2007)

61. M. Pavone, A. Sillanpaa, P. Cimino, O. Crescenzi, V. Barone, J. Phys. Chem. B. 110, 16189-16192 (2006)

62. M. Plato, H.J. Steinhoff, C. Wegener, J.T. Torring, A. Savitsky, K. Möbius, Mol. Phys. 100, 3711$3721(2002)$

63. A. Nalepa, K. Möbius, W. Lubitz, A. Savitsky, J. Magn. Reson. 242, 203-213 (2014)

64. G.I. Likhtenshtein, Spin labeling in biochemistry and biophysics, in Nitroxides. ed. by G.I. Likhtenshtein, J. Yamauchi, S.I. Nakatsuji, A.I. Smirnov, R. Tamura (Wiley-VCH, 2008), pp. 331-369

65. S. Schreier, J.C. Bozelli, N. Marín, R.F.F. Vieira, C.R. Nakaie, Biophys. Rev. 4, 45-66 (2012)

66. A.S. Kabankin, G.M. Zhidomirov, A.L. Buchacheko, J. Magn. Reson. 9, 199-204 (1973)

67. T. Otsuka, W. Motozaki, K. Nishikawa, K. Endo, J. Mol. Struct. 615, 147-151 (2002)

68. N.M. Atherton, Principles of Electron Spin Resonance (Ellis Horwood, 1993)

69. A.J. Schweiger, G. Jeschke, Principles of Pulse Electron Paramagnetic Resonance (Oxford University Press, 2001)

70. A. Savitsky, A.A. Dubinskii, M. Plato, Y.A. Grishin, H. Zimmermann, K. Möbius, J. Phys. Chem. B. 112, 9079-9090 (2008)

71. E. Reijerse, F. Lendzian, R. Isaacson, W. Lubitz, J. Magn. Reson. 214, 237-243 (2012)

72. K. Möbius, A. Savitsky, A. Schnegg, M. Plato, M. Fuchs, Phys. Chem. Chem. Phys. 7, 19-42 (2005) 
73. K. Möbius, A. Savitsky, High-Field EPR Spectroscopy on Proteins and Their Model Systems: Characterization of Transient Paramagnetic States (RSC Publishing, 2009)

74. E. Reijerse, P.P. Schmidt, G. Klihm, W. Lubitz, Appl. Magn. Reson. 31, 611 (2007)

75. J.V. Koleske, J.A. Faucher, Polym. Eng. Sci. 19, 716-721 (1979)

76. S.S.N. Murthy, S.K. Nayak, J. Chem. Phys. 99, 5362-5368 (1993)

77. S. Stoll, A. Schweiger, J. Magn. Reson. 178, 42-55 (2006)

78. F. Neese, WIREs Comput. Mol. Sci. 2, 73-78 (2012)

79. A. Schafer, H. Horn, R. Ahlrichs, J. Chem. Phys. 97, 2571-2577 (1992)

80. A. Savitsky, M. Plato, K. Möbius, Appl. Magn. Reson. 37, 415-434 (2010)

81. S.A. Dikanov, M.K. Bowman, J. Magn. Reson. A. 116, 125-128 (1995)

82. P. Cimino, M. Pavone, V. Barone, J. Phys. Chem. A. 111, 8482-8490 (2007)

83. C. Houriez, M. Masella, N. Ferre, J. Chem. Phys. 1, 133 (2010)

84. J. Heller, H. Elgabarty, B.L. Zhuang, D. Sebastiani, D. Hinderberger, J. Phys. Chem. B. 114, 74297438 (2010)

85. T.I. Smirnova, A.I. Smirnov, S.V. Paschenko, O.G. Poluektov, J. Am. Chem. Soc. 129, 3476 (2007)

86. D. Sezer, M.J. Prandolini, T.F. Prisner, Phys. Chem. Chem. Phys. 11, 6626-6637 (2009)

87. A. Alonso, O.R. Nascimento, M. Tabak, Nuovo Cimento Soc. Ital. Fis. D-Condens. Matter At Mol. Chem. Phys. Fluids Plasmas Biophys. 9, 227-245 (1987)

88. V.S. Kuts, T.S. Slipenyuk, É.P. Platonova, V.D. Pokhodenko, Theor. Exp. Chem. 13, 268-271 (1978)

89. G. Jeschke, Prog. Nucl. Magn. Reson. Spectrosc. 72, 42-60 (2013)

90. E. Ravera, C. Luchinat, G. Parigi, J. Magn. Reson. 264, 78-87 (2016)

91. M. Levien, M. Reinhard, M. Hiller, I. Tkach, M. Bennati, T. Orlando, Phys. Chem. Chem. Phys. 23, 4480-4485 (2021)

Publisher's Note Springer Nature remains neutral with regard to jurisdictional claims in published maps and institutional affiliations.

\section{Authors and Affiliations}

\section{Anton Savitsky ${ }^{1}$ (D) Anna Nalepa ${ }^{2} \cdot$ Taras Petrenko $^{3} \cdot$ Martin Plato $^{4}$. Klaus Möbius ${ }^{4} \cdot$ Wolfgang Lubitz $^{2}$ (D)}

Anton Savitsky

anton.savitsky@tu-dortmund.de

1 Faculty of Physics, Technical University Dortmund, Otto-Hahn-Str. 4a, 44227 Dortmund, Germany

2 Max-Planck-Institut für Chemische Energiekonversion, Stiftstr. 34-36, 45470 Mülheim (Ruhr), Germany

3 High Performance Computing Center Stuttgart (HLRS), Nobelstr. 19, 70550 Stuttgart, Germany

4 Fachbereich Physik, Freie Universität Berlin, Arnimallee 14, 14195 Berlin, Germany 